PHYSICAL REVIEW D 90, 095015 (2014)

\title{
Stop on top: SUSY parameter regions and fine-tuning constraints
}

\author{
Durmuş Ali Demir ${ }^{1, *}$ and Cem Salih Ün ${ }^{2, \dagger}$ \\ ${ }^{1}$ Department of Physics, İzmir Institute of Technology, IZTECH, TR35430 İzmir, Turkey \\ ${ }^{2}$ Department of Physics, Uluda $\breve{g}$ University, TR16059 Bursa, Turkey
}

(Received 14 July 2014; published 17 November 2014)

\begin{abstract}
We analyze minimal supersymmetric models in order to determine in what parameter regions with what amount of fine-tuning they are capable of accommodating the LHC-allowed top-stop degeneracy window. The stops must be light enough to enable Higgs naturalness yet heavy enough to induce a $125 \mathrm{GeV}$ Higgs boson mass. These two constraints imply a large mass splitting. By an elaborate scan of the parameter space, we show that the stop-on-top scenario requires at least $\Delta_{\mathrm{CMSSM}} \simeq \mathcal{O}\left(10^{4}\right)$ fine-tuning in the constrained minimal supersymmetric Standard Model (CMSSM). By relaxing the CMSSM parameter space with nonuniversal Higgs masses, we find that $\Delta_{\mathrm{NUHM} 1} \simeq \mathcal{O}\left(10^{4}\right)$. The CMSSM with a gravitino lightest supersymmetric particle works slightly better than the nonuniversal Higgs mass model. Compared to all these, the CMSSM with $\mu<0$ and nonuniversal gauginos yields a much smaller fine-tuning $\Delta_{\mu, g} \simeq \mathcal{O}(100)$. Our results show that the gaugino sector can pave the road toward a more natural stop-ontop scenario.
\end{abstract}

DOI: 10.1103/PhysRevD.90.095015

PACS numbers: 12.60.Jv, 14.65.Ha, 12.60.-i, 14.80.Ly

\section{INTRODUCTION}

A new bosonic resonance of mass about $125 \mathrm{GeV}$ has recently been discovered by ATLAS [1] and CMS [2] experiments at CERN. Even though the current analyses show that this new resonance exhibits properties very similar to the Standard Model (SM) Higgs boson, there is no doubt that the SM is not the final description of nature due to its drawbacks such as the gauge hierarchy problem [3] and absolute stability of the SM Higgs potential [4]. The $125 \mathrm{GeV}$ Higgs boson can give hints of new physics beyond the SM, and here supersymmetry (SUSY) stands out as one of the forefront candidates. The minimal supersymmetric extension of the SM (MSSM) can resolve the gauge hierarchy problem by invoking superpartners of the SM fields. Also, the lightest $C P$-even Higgs boson, one of the five physical Higgs states of the MSSM, exhibits very similar properties as the SM's Higgs boson in the decoupling limit [5]. In addition, imposing R-parity conservation, the MSSM gives a highly plausible candidate for the dark matter in the lightest supersymmetric particle (LSP).

Nevertheless, the LHC, with hadron smasher experiments, has brought very severe bounds on the color sector. Since there is no significant deviation in the Higgs production and decay properties with respect to the SM, and no significant signal of the supersymmetric particles, results from ATLAS and CMS have lifted the lower bounds on masses of gluinos and squarks in the first two generations up to $\sim 1.7 \mathrm{TeV}$ [6]. When squarks of the first two generations are heavy and decoupled, the bound on gluino

\footnotetext{
*demir@physics.iztech.edu.tr

†cemsalihun@uludag.edu.tr
}

mass is lowered depending on its decay channels. For instance, through gluino-mediated pair production of the third-generation squarks, gluinos of mass up to $\sim 1-1.3 \mathrm{TeV}$ are excluded depending on the final states $[6,7]$. Even though ATLAS and CMS results are presently not very severe for the third-generation squarks, stop masses in the range of $100-750 \mathrm{GeV}$ have been excluded for a massless LSP [8] (bounds on the stop mass are relaxed for a massive LSP). The stop decay channels $\tilde{t} \rightarrow t \tilde{\chi}_{1}^{0}$ and $\tilde{t} \rightarrow b \tilde{\chi}_{1}^{ \pm}$exclude stop masses up to $650 \mathrm{GeV}$ [9]. Moreover, the ATLAS collaboration has recently looked for the next-to-lightest supersymmetric particle (NLSP) stop via its decay mode to a charm quark and LSP, and the results have excluded stop masses up to $230 \mathrm{GeV}$ [10]. This channel also puts a lower bound on gluino mass as $m_{\tilde{g}} \gtrsim 1.1 \mathrm{TeV}[11]$.

These exclusion model-specific limits express nonobservation of light stops and gluinos at the LHC. The exclusion is not entire. The reason is that a small region with $m_{\tilde{t}_{1}} \lesssim 200 \mathrm{GeV}$ in the $m_{\tilde{\chi}_{1}^{0}}-m_{\tilde{t}_{1}}$ plane has not been excluded yet. Indeed, discrimination of $t \bar{t}$ and $\tilde{t}^{*}$ events with identical final states in this region is challenging [12], and the $\tilde{t}^{*}$ cross section stays in the error bar in the calculation of top pair production [13], which is measured to be [14]

$$
\sigma_{t \bar{t}}^{\sqrt{s}=8 \mathrm{TeV}}=241 \pm 2(\text { stat }) \pm 31 \text { (syst) } \pm 9 \text { (lumi) pb. }
$$

As has been well known for a long time, the Higgs boson mass is bounded from above by $M_{Z}$ at tree level in the MSSM, and hence one definitely needs to use radiative corrections in order to have $125 \mathrm{GeV}$ Higgs boson mass. Since the Yukawa couplings for the first two families are negligible, the third family stands out as the dominant 
source inducing such sizeable quantum contributions to Higgs boson mass. The sbottom contribution is proportional to the sbottom mixing parameter and $\mu \tan \beta$. However, strong bounds from vacuum stability on the $\mu \tan \beta$ term allow only a minor contribution from the sbottom sector [15]. Thus, the $125 \mathrm{GeV}$ Higgs boson largely constrains the stop sector. In the case of $m_{\tilde{t}_{L}} \simeq m_{\tilde{t}_{R}}$, the left- and right-handed stop masses are excluded up to $\sim 800 \mathrm{GeV}$. If one sets a hierarchy between the two stops $\left(m_{\tilde{t}_{L}} \ll m_{\tilde{t}_{R}}\right.$ or vice versa), then it becomes possible to have a light stop, while the other must weigh above $\sim 1 \mathrm{TeV}$ for moderate $\tan \beta$ [16]. This hierarchy necessitates a large stop mixing. Since the MSSM has many scalar fields, one may be concerned about color and/or charge breaking (CCB) minima that could occur in the case of large mixings at which some scalar fields may develop nonzero vacuum expectation values (VEVs). Even though large $m_{0}$ can ensure the absence of such minima [17], specifically the sfermions of the third family need a careful treatment, since their VEVs may cause tunneling into a deeper CCB minimum [18]. Among the MSSM scalars, stop has a special importance, since its nonzero VEV breaks $S U(3)_{c}$ and $U(1)_{\mathrm{em}}$ both. We thus check the vacuum stability for the constrained MSSM (CMSSM) benchmark points listed in the text. Apart from semianalytic estimates, the public code Vevacious [19] returns stable vacua for certain parameter regions accommodating light stops. The stability of the CMSSM points encourages us to conclude stability of more relaxed, less constrained SUSY models.

Returning to the large mixing, a $125 \mathrm{GeV}$ Higgs boson in the MSSM necessitates large splitting between the two stops, and this obviously contradicts with the naturalness domain $m_{\tilde{t}_{1}}, m_{\tilde{t}_{2}}, m_{\tilde{b}_{1}} \lesssim 500 \mathrm{GeV}$ [20] unless some extensions of the MSSM are considered [21]. From the naturalness point of view, one thus concludes that light stop regions in the MSSM need significant fine-tuning to yield the electroweak scale $\left(M_{\mathrm{EW}} \sim 100 \mathrm{GeV}\right)$ correctly. To analyze the amount of fine-tuning in allowed parameter regions, one can specifically focus on the Z-boson mass $\left(M_{\mathrm{Z}}=91.2 \mathrm{GeV}\right)$

$$
\frac{1}{2} M_{Z}^{2}=-\mu^{2}+\frac{\left(m_{H_{d}}^{2}+\Sigma_{d}^{d}\right)-\left(m_{H_{u}}^{2}+\Sigma_{u}^{u}\right) \tan ^{2} \beta}{\tan ^{2} \beta-1},
$$

which follows from the minimization of the MSSM Higgs potential [22] such that $\mu$ is the Higgsino Dirac mass, $\tan \beta=\left\langle H_{u}^{0}\right\rangle /\left\langle H_{d}^{0}\right\rangle, \Sigma_{u}^{u}$ and $\Sigma_{d}^{d}$ are radiative effects from the Higgs potential, and $m_{H_{u, d}}^{2}$ are the soft masses of the Higgs doublets $H_{u, d}$ that give mass to u-type and d-type fermions.

For quantifying the amount of fine-tuning associated with $M_{\mathrm{EW}}$, we use the measure defined in the recent work [23]. Namely, we introduce the electroweak fine-tuning

$$
\Delta_{\mathrm{EW}} \equiv \operatorname{Max}\left(C_{i}\right) /\left(M_{\mathrm{Z}}^{2} / 2\right),
$$

where

$$
C_{i} \equiv\left\{\begin{array}{l}
C_{H_{d}}=\left|m_{H_{d}}^{2} /\left(\tan ^{2} \beta-1\right)\right| \\
C_{H_{u}}=\left|m_{H_{u}}^{2} \tan ^{2} \beta /\left(\tan ^{2} \beta-1\right)\right| \\
C_{\mu}=\left|-\mu^{2}\right|
\end{array}\right.
$$

follow from the Higgs potential for which the parameters are evaluated at the electroweak scale.

The fine-tuning criterion $\Delta_{\mathrm{EW}}$ in (3) can be analyzed in comparison to the Barbieri-Giudice definition [24]

$$
\Delta_{\mathrm{BG}} \equiv \operatorname{Max}\left(B_{i}\right) /\left(M_{\mathrm{Z}}^{2} / 2\right),
$$

in which the coefficients

$$
B_{i}= \begin{cases}B_{H_{d}}=\left|m_{H_{d}}^{2}(\Lambda) /\left(\tan ^{2} \beta-1\right)\right|, & B_{\delta H_{d}}=\left|\delta m_{H_{d}}^{2} /\left(\tan ^{2} \beta-1\right)\right| \\ B_{H_{u}}=\left|m_{H_{u}}^{2}(\Lambda) /\left(\tan ^{2} \beta-1\right)\right|, & B_{\delta H_{u}}=\left|\delta m_{H_{u}}^{2} /\left(\tan ^{2} \beta-1\right)\right| \\ B_{\mu}=\left|-\mu^{2}(\Lambda)\right|, & B_{\delta \mu}=\left|-\delta \mu^{2}\right|\end{cases}
$$

are evaluated at high-energy scale $\Lambda$. Obviously, the electroweak fine-tuning in Eq. (3) can also be continued to the $\Lambda$ scale via renormalization group running and inclusion of the threshold corrections [23].

Note that in the calculation of $\Delta_{\mathrm{BG}} \delta m_{H_{u, d}}^{2}$ are considered separately in contrast to those in Eq. (3). In fact, $\Delta_{\mathrm{BG}}$ is calculated in terms of high-scale parameters such as $m_{H_{u, d}}^{2}(\Lambda)$ and $\mu(\Lambda)$ where $\Lambda$ denotes the highest energy scale up to which the model under concern is a valid effective field theory. In this approach, $\Delta_{\mathrm{BG}}$ contains information on the possible high-scale origin of the fine-tuning. Gravity-mediated supersymmetric theories such as minimal supergravity (mSUGRA) are, in general, assumed to be valid up to the grand unified theory (GUT) scale, and hence $\Delta_{\mathrm{BG}}$ is calculated with terms that are normalized at $\Lambda=M_{\mathrm{GUT}}$. In such models, $B_{\delta H_{u^{\prime}}}$ becomes dominant because of large logarithms. Also, $m_{H_{u}}^{2}$ needs a significant contribution, $\delta m_{H_{u}}^{2}$, since it is required to evolve to negative values from its high-scale values as required by radiative electroweak symmetry breaking (REWSB) [25].

The essential observation is that light stop can hide in the top signal in the region with $m_{\tilde{t}_{1}} \lesssim 200 \mathrm{GeV}$. The reported 
top results can thus contain the stop signal within the exclusion limits. In the present paper, our goal is to determine SUSY parameter regions accommodating light stops. In doing this, we consider CMSSM and SUSY GUTs and investigate their "light stop regions" by taking into account bounds from $125 \mathrm{GeV}$ Higgs boson, $B$ physics, and cold dark matter. We also give the results for naturalness in terms of $\Delta_{\mathrm{EW}}$ and $\Delta_{\mathrm{BG}}$ in a general fashion. Besides these, we provide the masses of supersymmetric particles that allow computation of the fine-tuning measure. In our approach, the fine-tuning is an indicator of the fact that the model has some missing mechanism that would produce the desired result naturally, and its amount shows how effective the missing mechanism is. In this context, we use no prejudice or presetting on the amount of fine-tuning, and we do not apply it as a constraint. To have better results for the fine-tuning, one can consider extensions of the MSSM [26]. Despite different models considered, these exclusive studies more or less agree on that an acceptable amount of fine-tuning $\left(\Delta_{\mathrm{EW}} \lesssim 10^{3}\right)$ constrains the stop mass from below at about $500 \mathrm{GeV}$. In other words, the models with acceptable fine-tuning exclude the regions that yield stops nearly degenerate with the top quark. The mixing between the stop quarks is proportional to $A_{t}-\mu \cot \beta$, and the $\mu$ term should be in a range in order to have such light stops, and this range leads to a highly fine-tuned model. In this context, one can consider to reverse the effect of $\mu$ in the mixing of stops by considering the negative values for $\mu$, since the minimization of the Higgs potential allows $\mu<0$ as well as $\mu>0$. In our study, we consider several models and determine the regions in their parameter spaces that yield the stop mass nearly degenerate with the top quark. In each case, we explicitly reveal the requisite fine-tuning by evaluating $\Delta_{\mathrm{EW}}$ and $\Delta_{\mathrm{BG}}$. Last but not least, we consider the nonuniversal gaugino masses at the GUT scale and show that a model with $\mu<0$ and nonuniversal gaugino masses can provide a more natural model for the light stop region.

The outline of the paper is as follows. In Sec. II, we describe scanning procedure and various experimental bounds to be imposed. Section III contains our results for the CMSSM. Section IV is devoted to nonuniversal Higgs mass (NUHM1) models. Section V discusses the CMSSM with the gravitino LSP. Section VI deals with nonuniversal gaugino masses and negative $\mu$. Finally, we conclude in Sec. V.

\section{SCANNING PROCEDURE AND EXPERIMENTAL CONSTRAINTS}

There are several publicly available numerical codes and event generators specialized for specific purposes. For instance, SoftSUSY [27] and SPheno [28] calculate the supersymmetric mass spectrum, where one can use Feynhiggs [29] for properties of the Higgs boson, SuFla [30] for the B-physics observables, MICROMEGAs [31],
DARKSUSY [32], and AstroFit [33] for the dark matter features. There are also some frameworks such as Fittino [34] and Mastercode [35] performing Markov chain Monte Carlo (MCMC) scans that incorporate several numerical codes and event generators. In our study, we employ the ISAJET 7.84 package [36] to perform random scans over the parameter space. In this package, the weak scale values of gauge and third-generation Yukawa couplings are evolved to $M_{\mathrm{GUT}}$ via renormalization group equations (RGEs) in the $\overline{\mathrm{DR}}$ regularization scheme. We do not strictly enforce the gauge unification condition $g_{1}=$ $g_{2}=g_{3}$ at $M_{\mathrm{GUT}}$, since a few percent deviation from unification can be assigned to unknown GUT-scale threshold corrections [37]. With the boundary conditions given at $M_{\mathrm{GUT}}$, all the soft supersymmetry breaking (SSB) parameters, along with the gauge and Yukawa couplings, are evolved back to the weak scale $M_{\mathrm{Z}}$.

In renormalization group evolution of Yukawa couplings, the SUSY threshold corrections [38] are taken into account at the common scale $M_{\mathrm{SUSY}}=\sqrt{m_{\tilde{t}_{L}} m_{\tilde{t}_{R}}}$. The entire parameter set is iteratively run between $M_{Z}$ and $M_{\mathrm{GUT}}$ using the full two-loop RGEs until a stable solution is obtained. To better account for leading-log corrections, one-loop step beta functions are adopted for gauge and Yukawa couplings, and the SSB parameters $m_{i}$ are extracted from RGEs at appropriate scales $m_{i}=m_{i}\left(m_{i}\right)$. The RGE-improved one-loop effective potential is minimized at an optimized scale $M_{\text {SUSY }}$, which effectively accounts for the leading two-loop corrections. Full oneloop radiative corrections are incorporated for all sparticle masses.

While scanning, we perform MCMC scans over the parameter spaces of the models we analyze and calculate the low-energy observables by using ISAJET. We also follow the $\chi^{2}$ analysis by defining

$$
\chi^{2}=\exp \left[-\frac{\left(m_{\tilde{t}}-m_{t}\right)^{2}}{\sigma^{2}}\right]
$$

where $m_{\tilde{t}}$ is the stop mass and $m_{t}=173.3 \mathrm{GeV}$ is the top quark mass $[39,40]$. Note that our results are not too sensitive to variation in the value of $m_{t}$ within $1 \sigma-2 \sigma$ [41].

We employ the Metropolis-Hasting algorithm as described in Ref. [42] and require all points to satisfy REWSB. The REWSB requirement is a crucial theoretical constraint on the parameter space [43]. After collecting data, we subsequently impose the mass bounds [44] and B-physics constraints, the stop-top degeneracy band $\left(\Delta m_{\tilde{t} t} \leq 30 \mathrm{GeV}\right)$, and the WMAP bound on the relic density of the neutralino LSP. The B-physics observables and relic density of neutralino LSP are calculated by the use of IsaTools [45,46]. The experimental constraints imposed in our data can be summarized as follows [47-50]: 


$$
\begin{gathered}
m_{h}=(123-127) \mathrm{GeV} \\
m_{\tilde{g}} \geq 1 \mathrm{TeV} \\
0.8 \times 10^{-9} \leq \mathrm{BR}\left(B_{s} \rightarrow \mu^{+} \mu^{-}\right) \leq 6.2 \times 10^{-9}(2 \sigma) \\
2.99 \times 10^{-4} \leq \mathrm{BR}(b \rightarrow s \gamma) \leq 3.87 \times 10^{-4}(2 \sigma) \\
0.15 \leq \frac{\mathrm{BR}\left(B_{u} \rightarrow \tau \nu_{\tau}\right)_{\mathrm{MSSM}}}{\mathrm{BR}\left(B_{u} \rightarrow \tau \nu_{\tau}\right)_{\mathrm{SM}}} \leq 2.41(3 \sigma) \\
0.0913 \leq \Omega_{\mathrm{CDM}} h^{2}(\mathrm{WMAP}) \leq 0.1363(5 \sigma)
\end{gathered}
$$

We display the mass bounds on the Higgs boson $[1,2]$ and gluino [6] because they have changed since the LEP era. We take the lower bound on gluino mass as $m_{\tilde{g}} \geq$ $1 \mathrm{TeV}$ all over the parameter space since the exclusion curve excludes the gluino of mass less than $1 \mathrm{TeV}$ for the LSP of mass less than $300 \mathrm{GeV}$. Besides these constraints, we require our solution to do no worse than the SM in prediction of the muon anomalous magnetic moment (muon $g-2$ ).

\section{CMSSM}

In this section, we study the CMSSM parameter space to determine under what conditions it can accommodate stoptop degeneracy. In doing this, we scan their parameter spaces to determine viable regions and also compute the naturalness of those regions.

In this section, we scan CMSSM parameter space within the ranges
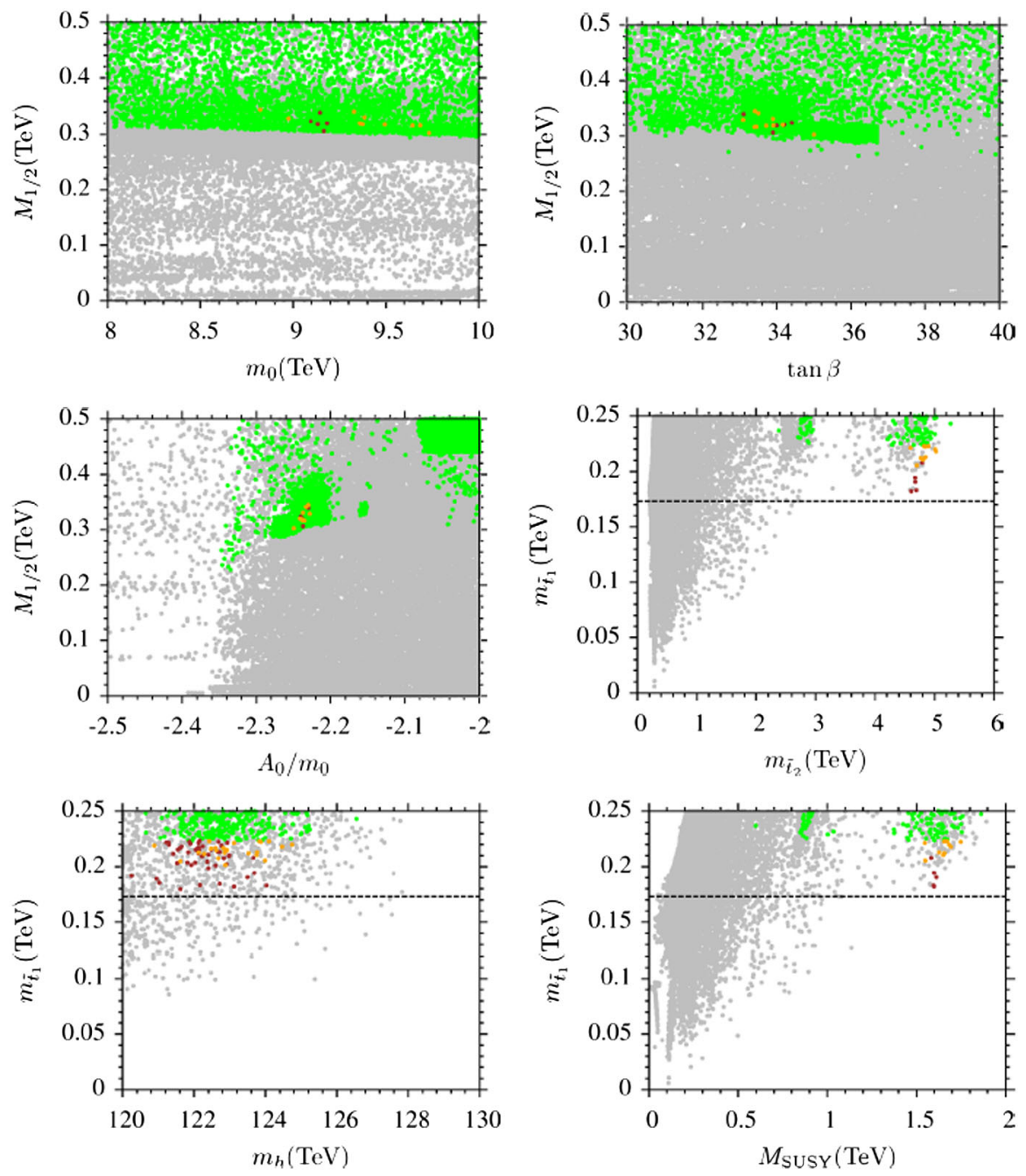

FIG. 1 (color online). Plots in $M_{1 / 2}-m_{0}, M_{1 / 2}-\tan \beta, M_{1 / 2}-A_{0} / m_{0}, m_{\tilde{t}_{1}}-m_{\tilde{t}_{2}}, m_{\tilde{t}_{1}}-M_{\mathrm{SUSY}}$, and $m_{\tilde{t}_{1}}-m_{h}$ planes for the CMSSM with the neutralino LSP. All points are consistent with REWSB and the neutralino LSP. Green points satisfy mass bounds and B-physics bounds mentioned in Sec. II. The red points within the green are the ones that satisfy $\Delta m_{\tilde{t} t} \leq 50 \mathrm{GeV}$ where $\Delta m_{\tilde{t} t} \equiv m_{\tilde{t}_{1}}-m_{t}$. The orange points are a subset of red points and satisfy the WMAP bound on the relic abundance of the neutralino within $5 \sigma$. The dashed line corresponds to top quark mass $m_{t}=173.3 \mathrm{Gev}$. 

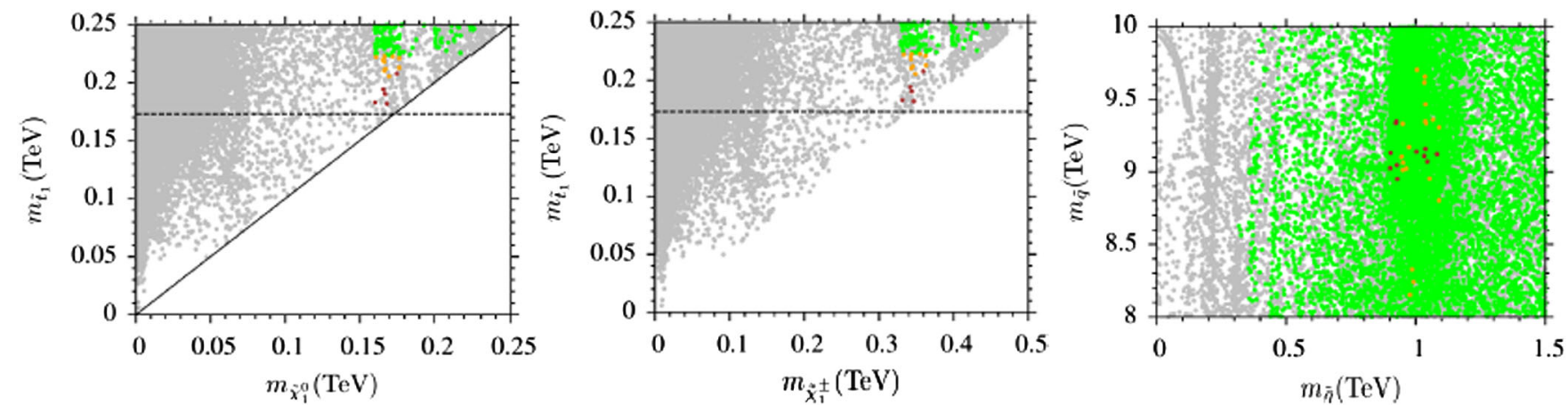

FIG. 2 (color online). The sparticle spectrum in $m_{\tilde{t}_{1}}-m_{\tilde{\chi}_{1}^{0}}, m_{\tilde{t}_{1}}-m_{\tilde{\chi}_{1}^{ \pm}}$, and $m_{\tilde{q}}-m_{\tilde{g}}$ planes for the CMSSM with the neutralino LSP. The color coding is the same as in Fig. 1, except the lower mass bound on gluino is not applied in the $m_{\tilde{q}}-m_{\tilde{g}}$ panel.

$$
\begin{gathered}
0 \leq m_{0} \leq 20 \mathrm{TeV} \\
0 \leq M_{1 / 2} \leq 5 \mathrm{TeV} \\
-3 \leq A_{0} / m_{0} \leq 3 \\
2 \leq \tan \beta \leq 60
\end{gathered}
$$

where $m_{0}$ is the universal SSB mass term for all scalars including $H_{u}$ and $H_{d}$ of the MSSM and $M_{1 / 2}$ is the universal SSB gaugino mass term. The $A_{0}$ is a SSB trilinear scalar interaction term, and $\tan \beta$ is the ratio of vacuum expectation values of the MSSM Higgs fields.

Figure 1 displays the plots in $M_{1 / 2}-m_{0}, M_{1 / 2}-\tan \beta$, $M_{1 / 2}-A_{0} / m_{0}, \quad m_{\tilde{t}_{1}}-m_{\tilde{t}_{2}}, \quad m_{\tilde{t}_{1}}-M_{\mathrm{SUSY}}$, and $m_{\tilde{t}_{1}}-m_{h}$ planes. All points are consistent with REWSB and the neutralino LSP. Green points satisfy mass bounds and B physics mentioned in Sec. II. Red points form a subset of green, and they satisfy $\Delta m_{\tilde{t} t} \leq 50 \mathrm{GeV}$ where $\Delta m_{\tilde{t} t} \equiv m_{\tilde{t}_{1}}-m_{t}$. Orange points are a subset of red points satisfying the WMAP bound on the relic abundance of the neutralino within $5 \sigma$. The dashed line corresponds to top quark mass $m_{t}=173.3 \mathrm{GeV}$. Results displayed in $M_{1 / 2}-m_{0}, M_{1 / 2}-\tan \beta$, and $M_{1 / 2}-A_{0} / m_{0}$ panels show that the degeneracy between top quark and its supersymmetric partner can be realized in a very small region with $m_{0} \sim 9 \mathrm{TeV}, M_{1 / 2} \sim 300-350 \mathrm{GeV}, \tan \beta \sim 34$, and $A_{0} / m_{0} \sim-2.2$. The large mixing seen in the $M_{1 / 2}-A_{0} / m_{0}$ plane leads to a huge mass difference between the two stop mass eigenstates, $\tilde{t}_{1}$ and $\tilde{t}_{2}$. It is seen from the $m_{\tilde{t}_{1}}-m_{\tilde{t}_{2}}$
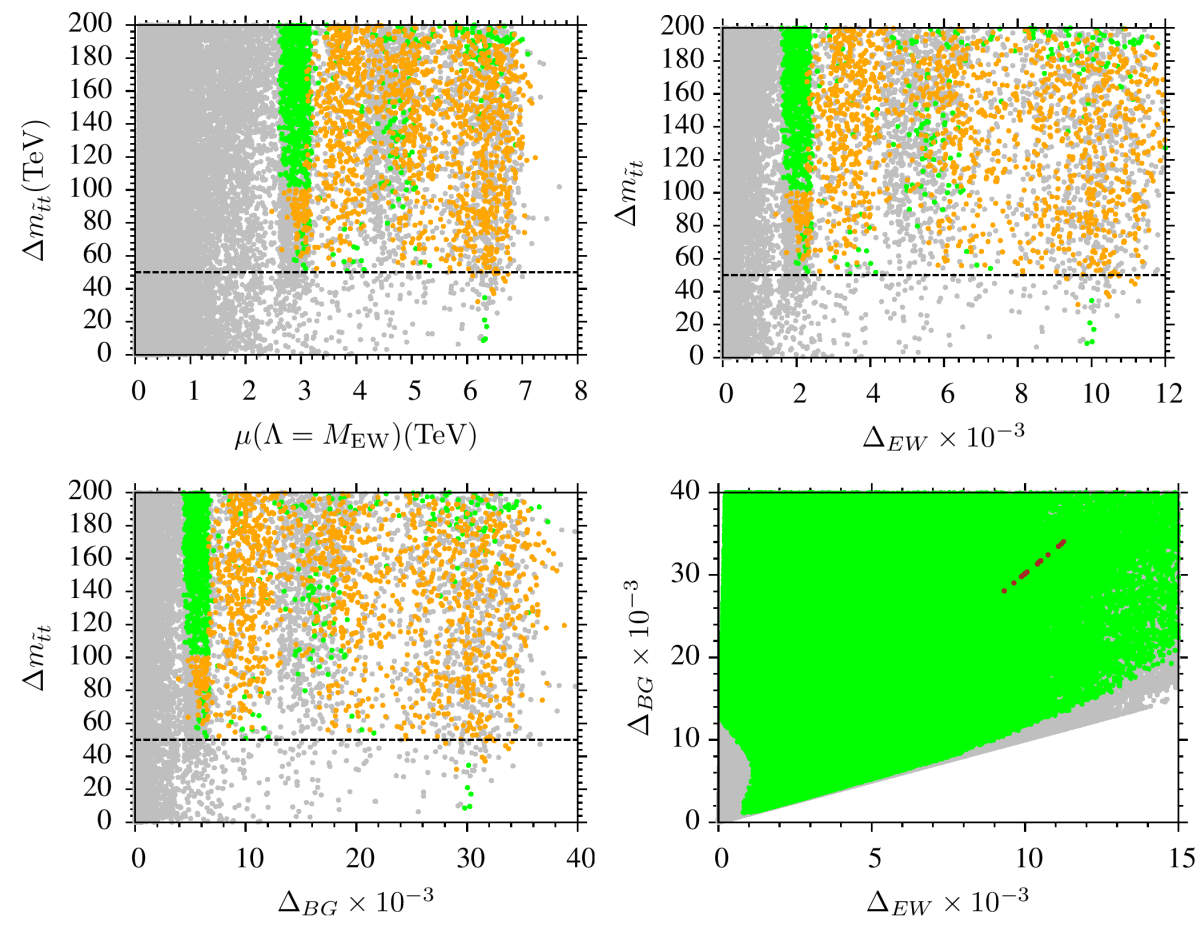

FIG. 3 (color online). Fine-tuning plots in $\Delta m_{\tilde{t} t}-\mu, \Delta m_{\tilde{t} t}-\Delta_{\mathrm{EW}}, \Delta m_{\tilde{t} t}-\Delta_{\mathrm{BG}}$, and $\Delta_{\mathrm{BG}}-\Delta_{\mathrm{EW}}$ planes for the CMSSM with the neutralino LSP. The color coding is the same as Fig. 1, except that $\Delta m_{\tilde{t} t} \leq 50$ is not applied in the $\Delta m_{\tilde{t} t}-\mu, \Delta m_{\tilde{t} t}-\Delta \mathrm{EW}$, and $\Delta m_{\tilde{t} t}-\Delta_{\mathrm{BG}}$ panels; a guideline is rather used to indicate $\Delta m_{\tilde{t} t}=50$. 
plane that the heavier stop eigenstate has a mass about 4-5 TeV, while the lighter one stays close to the top quark. This large mixing is also required to satisfy the constraint of the $125 \mathrm{GeV}$ Higgs boson. The $m_{\tilde{t}_{1}}-m_{h}$ shows that it is possible to find solutions with the Higgs boson of mass about $124 \mathrm{GeV}$. Note that the Higgs mass is calculated in ISAJET with an approximate error of about $3 \mathrm{GeV}$ arising from theoretical uncertainties in calculation of the minimum of the scalar potential and experimental uncertainties in the values of $m_{t}$ and $\alpha_{s}$. From the $m_{\tilde{t}_{1}}-M_{\mathrm{SUSY}}$ plot, the SUSY decoupling scale reads $M_{\mathrm{SUSY}} \simeq 1.5 \mathrm{TeV}$, and it provides the desired solution for the gauge hierarchy problem within the $\mathrm{TeV}$-scale SUSY. It is true that the orange points form a tiny subset; however, once model parameters are fixed by them, the resulting model gives a viable SUSY description because the stop is sitting on top quark.

The spectrum for other SUSY particles is represented in the $m_{\tilde{t}_{1}}-m_{\tilde{\chi}_{1}^{0}}, m_{\tilde{t}_{1}}-m_{\tilde{\chi}_{1}^{ \pm}}$, and $m_{\tilde{q}}-m_{\tilde{g}}$ planes of Fig. 2 . The color coding is the same as Fig. 1, except the lower mass bound on the gluino is not applied in the $m_{\tilde{q}}-m_{\tilde{g}}$ panel. In the light stop region, it is also found that the lightest stop is almost degenerate with the LSP neutralino of mass about $160 \mathrm{GeV}$, as seen from the $m_{\tilde{t}_{1}}-m_{\tilde{\chi}_{1}^{0}}$ panel. The solutions with $m_{\tilde{\chi}_{1}^{0}} \lesssim 150 \mathrm{GeV}$ are not consistent with the experimental constraints mentioned in Sec. II. Similarly, the lightest chargino is of mass about $300 \mathrm{GeV}$ in the same region. The $m_{\tilde{q}}-m_{\tilde{g}}$ plane reveals important results for the color sector obtained in the light stop region. The squarks of the first generations are found to be of mass about $9 \mathrm{TeV}$, which is beyond the exclusion limit. On the other hand, the gluino weighs slightly above $1 \mathrm{TeV}$.

Figure 3 shows the results for the fine-tuning calculated for the light stop region in the $\Delta m_{\tilde{t} t}-\mu, \Delta m_{\tilde{t} t}-\Delta_{\mathrm{EW}}$, $\Delta m_{\tilde{t} t}-\Delta_{\mathrm{BG}}$, and $\Delta_{\mathrm{BG}}-\Delta_{\mathrm{EW}}$ planes. The color coding is the same as Fig. 1 except that $\Delta m_{\tilde{t} t} \leq 50$ is not applied in the $\Delta m_{\tilde{t} t}-\mu, \Delta m_{\tilde{t} t}-\Delta_{\mathrm{EW}}$, and $\Delta m_{\tilde{t} t}-\Delta_{\mathrm{BG}}$ panels; a guideline is rather used to indicate $\Delta m_{\tilde{t} t}=50$. The fact that this region needs a large mixing between the stops results in a large SSB trilinear $A_{t}$ term, and it leads to a significant fine-tuning as seen from the plots of Fig. 3. The $\Delta m_{\tilde{t} t}-\mu$ plane shows that $\mu(\Lambda=$ weak $) \simeq 3 \mathrm{TeV}$ for $\Delta m_{\tilde{t} t} \simeq 50$, and it raises up to $6 \mathrm{TeV}$, if one seeks for $m_{\tilde{t} t} \leq 50$. Similarly, $\Delta_{\mathrm{EW}} \simeq 2000(0.05 \%)$ and $\Delta_{\mathrm{BG}} \simeq$ $6000(0.017 \%)$ for $\Delta m_{\tilde{t t}} \simeq 50$, while $\Delta_{\mathrm{EW}} \simeq 9000$ $(0.012 \%)$ and $\Delta_{\mathrm{BG}} \simeq 30000(0.003 \%)$ for $\Delta m_{\tilde{t} t} \leq 50$. The $\Delta_{\mathrm{BG}}-\Delta_{\mathrm{EW}}$ plane summarizes the results obtained for the fine-tuning.

Another important constraint comes from the WMAP9 searches for the dark matter. A mass difference up to $20 \%$ between the NLSP stop and LSP neutralino is excluded in order to satisfy the WMAP bound on the relic abundance of the LSP neutralino for $m_{\tilde{\chi}_{1}^{0}} \lesssim 200 \mathrm{GeV}$ [51]. In our results for CMSSM, there is no solution that satisfies both the WMAP bound and $\Delta m_{\tilde{t} t} \leq 30 \mathrm{GeV}$. We also illustrate our
TABLE I. Benchmark points with $m_{\tilde{t}_{1}} \simeq m_{t}$ for the CMSSM with the neutralino LSP. Masses are given in $\mathrm{GeV}$ units. All points are chosen as to be consistent with mass and B-physics constraints. Point 1 displays a solution with the best degeneracy between stop and top quarks. Point 2 depicts a solution consistent with WMAP bounds on the relic abundance of the LSP neutralino within $5 \sigma$. Point 3 yields a $126 \mathrm{GeV}$ Higgs boson solution with the least mass separation between the stop and top quark. These points do not generate or tunnel to a deeper CCB minima.

\begin{tabular}{lccc}
\hline \hline & Point 1 & Point 2 & Point 3 \\
\hline$m_{0}$ & 9165 & 8975 & 9460 \\
$M_{1 / 2}$ & 305.9 & 328.9 & 308.7 \\
$\tan \beta$ & 33.9 & 33.1 & 31.0 \\
$A_{0} / m_{0}$ & -2.24 & -2.23 & -2.21 \\
$m_{t}$ & 173.3 & 173.3 & 173.3 \\
$\mu$ & 6134 & 6008 & 6312 \\
$\Delta a_{\mu}$ & $0.50 \times 10^{-11}$ & $0.39 \times 10^{-10}$ & $0.42 \times 10^{-11}$ \\
$m_{h}$ & 124.1 & 124 & 126.1 \\
$m_{H}$ & 6922 & 7049 & 8041 \\
$m_{A}$ & 6878 & 7004 & 7989 \\
$m_{H^{ \pm}}$ & 6923 & 7050 & 8042 \\
$m_{\tilde{\chi}_{1,2}^{0}}$ & $\mathbf{1 6 0 . 3 , 3 2 8 . 2}$ & $\mathbf{1 6 9 . 6 , 3 4 5 . 9}$ & $\mathbf{1 6 1 . 1 , 3 2 9 . 2}$ \\
$m_{\tilde{\chi}_{3,4}^{0}}$ & 6156,6156 & 6032,6032 & 6343,6344 \\
$m_{\tilde{\chi}_{1,2}^{ \pm}}$ & $\mathbf{3 3 1 . 2 , 6 1 7 1}$ & $\mathbf{3 4 9 . 1 , 6 0 4 7}$ & $\mathbf{3 3 2 . 3 , 6 3 6 1}$ \\
$m_{\tilde{g}}$ & $\mathbf{1 0 0 2}$ & $\mathbf{1 0 5 4}$ & $\mathbf{1 0 1 0}$ \\
$m_{\tilde{u}_{L, R}}$ & 9139,9172 & 8953,8980 & 9430,9460 \\
$m_{\tilde{t}_{1,2}}$ & $182.9,4696$ & $205.4,4705$ & $213.4,5192$ \\
$m_{\tilde{d}_{L, R}}$ & 9139,9172 & 8953,8985 & 9431,9465 \\
$m_{\tilde{b}_{1,2}}$ & 4803,6648 & 4813,6660 & 5311,7355 \\
$m_{\tilde{\nu}_{e, \mu}}$ & 9167 & 8977 & 9462 \\
$m_{\tilde{\nu}_{\tau}}$ & 8045 & 7925 & 8482 \\
$m_{\tilde{e}_{L, R}}$ & 9156,9159 & 8967,8969 & 9450,9454 \\
$m_{\tilde{\tau}_{1,2}}$ & 6855,8076 & 6819,7955 & 7483,8514 \\
$\sigma_{\mathrm{SI}}(\mathrm{pb})$ & $0.11 \times 10^{-11}$ & $0.11 \times 10^{-11}$ & $0.76 \times 10^{-12}$ \\
$\sigma_{\mathrm{SD}}(\mathrm{pb})$ & $0.37 \times 10^{-10}$ & $0.40 \times 10^{-10}$ & $0.32 \times 10^{-10}$ \\
$\Omega_{h^{2}}$ & 0.03 & 0.104 & 0.18 \\
$\Delta_{\mathrm{EW}}$ & 10014 & 9633 & 10691 \\
$\Delta_{\mathrm{BG}}$ & 30244 & 29034 & 32248 \\
\hline \hline & & & \\
\hline & & & \\
\hline & & & \\
\hline
\end{tabular}

results for the CMSSM in Table I. Masses are given in $\mathrm{GeV}$ units. All points are chosen so as to be consistent with mass and B-physics constraints. Point 1 displays a solution with the best degeneracy between stop and top quarks. As stated above, relic abundance of the LSP neutralino is too low for point 1 to satisfy the WMAP bound. Point 2 depicts a solution consistent with the WMAP bound on the relic abundance of the LSP neutralino within $5 \sigma$. The mass difference between the stop and neutralino is about $32 \mathrm{GeV}$ for point 2. Point 3 yields a $126 \mathrm{GeV}$ Higgs boson solution with the least mass separation between stop and top quarks. We checked by using the public code Vevacious [19] that point 1 , point 2 , and point 3 yield stable vacua.

\section{NUHM1}

Having found that the CMSSM is severely fine-tuned in producing the stop-top degeneracy region, we now start 

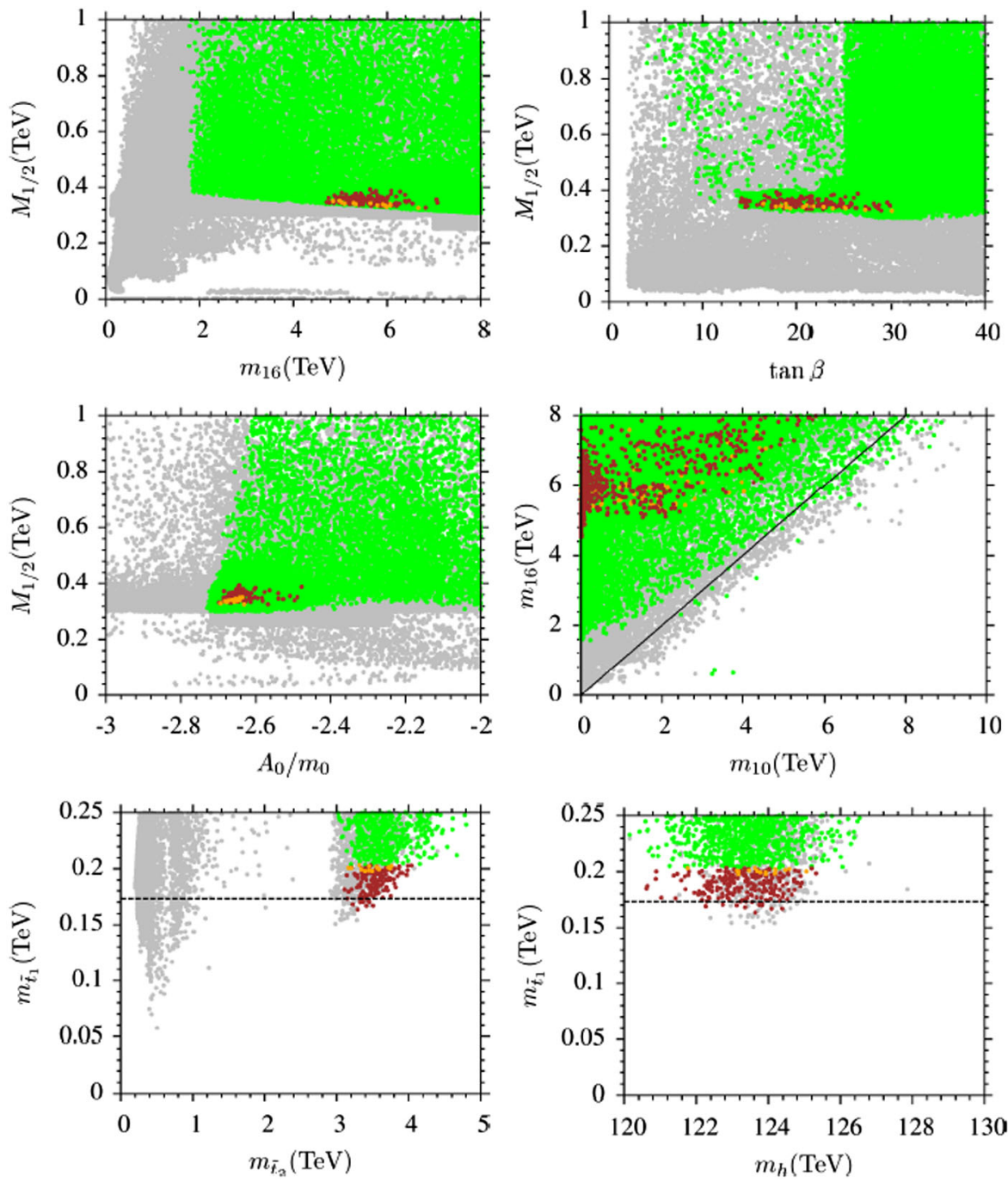

FIG. 4 (color online). Plots in the $M_{1 / 2}-m_{16}, M_{1 / 2}-\tan \beta, M_{1 / 2}-A_{0} / m_{16}, m_{16}-m_{10}, m_{\tilde{t}_{1}}-m_{\tilde{t}_{2}}, m_{\tilde{t}_{1}}-M_{\mathrm{SUSY}}$, and $m_{\tilde{t}_{1}}-m_{h}$ planes for NUHM1 with the neutralino LSP. The color coding is the same as Fig. 1, except that $\Delta m_{\tilde{t} t} \leq 30 \mathrm{GeV}$ is applied in the red region. The solid line in the $m_{16}-m_{10}$ plane corresponds to the CMSSM solution for which $m_{16}=m_{10}=m_{0}$.

looking for extensions of the CMSSM where fine-tuning is lower. We start our search with the CMSSM with nonunivseral Higgs masses at the unification scale. In other words, we relax the CMSSM setup by separating the SSB mass term for the MSSM Higgs fields from the one for the remaining matter scalars. The results displayed here are obtained from scanning over the parameter space

$$
\begin{gathered}
0 \leq m_{16} \leq 20 \mathrm{TeV} \\
0 \leq m_{10} \leq 20 \mathrm{TeV} \\
0 \leq M_{1 / 2} \leq 5 \mathrm{TeV} \\
-3 \leq A_{0} / m_{0} \leq 3 \\
2 \leq \tan \beta \leq 60
\end{gathered}
$$

where $m_{16} \equiv m_{0}$ and $m_{10}$ are the SSB mass terms for the matter scalars and the Higgs fields, respectively. All other parameters are the same as in the previous subsection.
Figure 4 displays the scan results in the $M_{1 / 2}-m_{16}$, $M_{1 / 2}-\tan \beta, \quad M_{1 / 2}-A_{0} / m_{16}, \quad m_{16}-m_{10}, \quad m_{\tilde{t}_{1}}-m_{\tilde{t}_{2}}$, $m_{\tilde{t}_{1}}-M_{\mathrm{SUSY}}$, and $m_{\tilde{t}_{1}}-m_{h}$ planes. The color coding is the same as Fig. 1, except that now the red region corresponds to $\Delta m_{\tilde{t} t} \leq 30 \mathrm{GeV}$ (which is not reachable in the CMSSM domain). The solid line in the $m_{16}-m_{10}$ plane corresponds to the region in which $m_{16}=m_{10}$ (the CMSSM solution). While we have a very narrow range for the SSB gaugino mass term $\left(M_{1 / 2} \sim 400 \mathrm{GeV}\right)$, compared to the CMSSM regions, the ranges for the other fundamental parameters become slightly wider because now $m_{16} \sim 4.5-7.5 \mathrm{TeV}, \quad m_{10} \lesssim 5 \mathrm{TeV}, \quad \tan \beta \sim 14-30, \quad$ and $A_{0} / m_{16} \sim-2.7--2.4$. Note that the SSB masses for the matter scalars is shifted back to $6 \mathrm{TeV}$, while it is strictly $\sim 9 \mathrm{TeV}$ in the CMSSM. As we can see from the $m_{16}-m_{10}$ panel, light Higgs masses at the GUT scale favor light stops at the low scale. On the other hand, a large mixing between left- and right-handed stops can occur because of large $A_{t}$ 

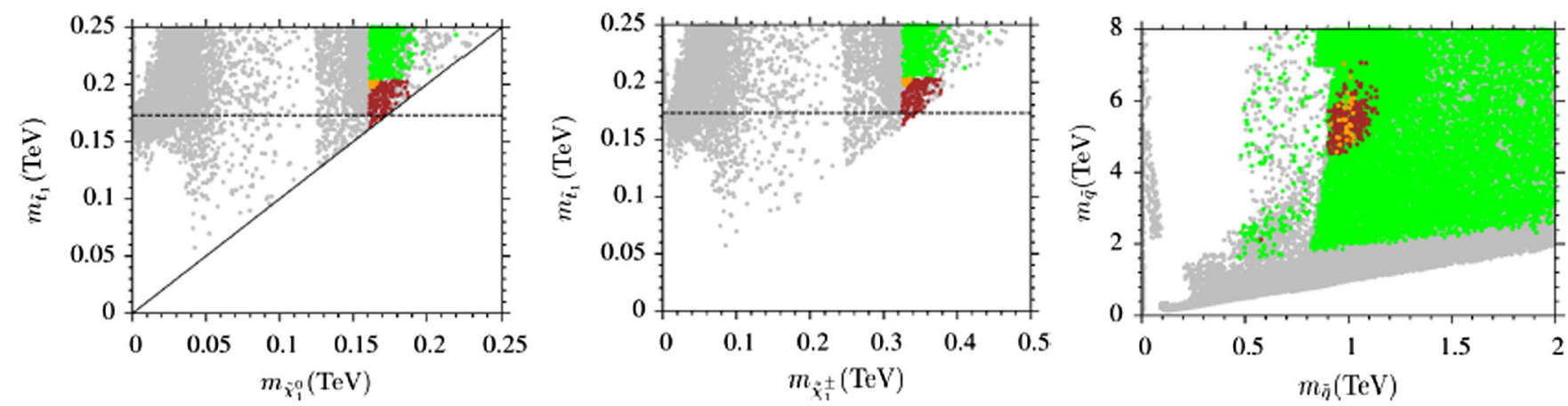

FIG. 5 (color online). SUSY spectrum in the $m_{\tilde{t}_{1}}-m_{\tilde{\chi}_{1}^{0}}, m_{\tilde{t}_{1}}-m_{\tilde{\chi}_{1}^{ \pm}}$, and $m_{\tilde{q}}-m_{\tilde{g}}$ planes for NUHM1 with the neutralino LSP. The color coding is the same as Fig. 4, except the lower mass bound on the gluino is not applied in the $m_{\tilde{q}}-m_{\tilde{g}}$ panel.

values, and the heavier stop is found to be of mass about 3-4 TeV. Also, the $m_{\tilde{t}_{1}}-m_{h}$ panel shows that it is possible to find the SM-like Higgs boson of mass up to $\sim 126 \mathrm{GeV}$.

We depict the results for the SUSY spectrum in the $m_{\tilde{t}_{1}}-m_{\tilde{\chi}_{1}^{0}}, m_{\tilde{t}_{1}}-m_{\tilde{\chi}_{1}^{ \pm}}$, and $m_{\tilde{q}}-m_{\tilde{g}}$ planes of Fig. 5. The color coding is the same as in Fig. 4 except that the lower mass bound on the gluino is not applied in the $m_{\tilde{q}}-m_{\tilde{g}}$ panel. Despite similar results to those obtained for the CMSSM, in the NUHM1 framework, it is possible to have a solution with $m_{\tilde{t}_{1}} \lesssim m_{t}$. We have an extreme degeneracy between the stop and neutralino, and hence neutralino relic abundance is too low as stated in the previous section. The $m_{\tilde{t}_{1}}-m_{\tilde{\chi}_{1}^{ \pm}}$panel indicates the chargino mass range $\sim 300-400 \mathrm{GeV}$. Squarks of the first generations have mass $\gtrsim 4 \mathrm{TeV}$, while the gluinos are found to be of mass $1-1.2 \mathrm{TeV}$, as seen in the $m_{\tilde{q}}-m_{\tilde{g}}$ plane.

We represent the results for the fine-tuning in $\Delta m_{\tilde{t} t}-\mu$, $\Delta m_{\tilde{t} t}-\Delta_{\mathrm{EW}}, \Delta m_{\tilde{t} t}-\Delta_{\mathrm{BG}}$, and $\Delta_{\mathrm{BG}}-\Delta_{\mathrm{EW}}$ planes of Fig. 6 . The color coding is the same as Fig. 3. The results are similar to those obtained for the CMSSM. $\mu(\Lambda=$ weak) $\sim 6-8 \mathrm{TeV}$ and $\Delta_{\mathrm{EW}} \sim 8000-12000 \quad(0.012 \%-$ $0.008 \%$ ) for $\Delta m_{\tilde{t} t} \leq 30$, while we observe an improvement in $\Delta_{\mathrm{BG}}$ as $\sim 8000-16000(0.012 \%-0.06 \%)$ compared to the CMSSM with the neutralino LSP.

Finally, we represent some benchmark points that exemplify our results obtained for NUHM1 in Table II. Masses are given in $\mathrm{GeV}$ units, and all points are chosen so as to be consistent with mass and B-physics constraints.
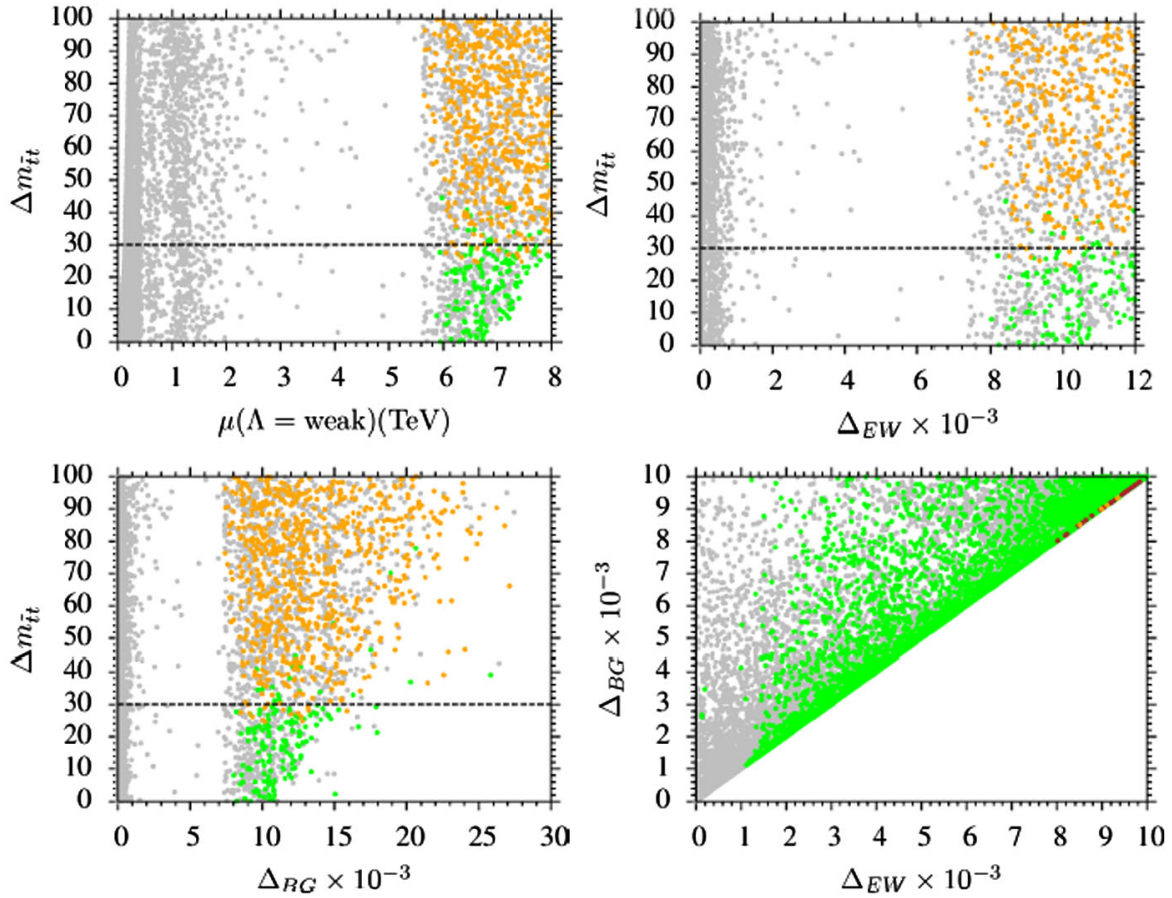

FIG. 6 (color online). Fine-tuning plots in the $\Delta m_{\tilde{t} t}-\mu, \Delta m_{\tilde{t} t}-\Delta_{\mathrm{EW}}, \Delta m_{\tilde{t} t}-\Delta_{\mathrm{BG}}$, and $\Delta_{\mathrm{BG}}-\Delta_{\mathrm{EW}}$ planes for NUHM1 with the neutralino LSP. The color coding is the same as Fig. 3. 
TABLE II. Benchmark points with $m_{\tilde{t}_{1}} \simeq m_{t}$ in the NUHM1 with the neutralino LSP. Masses are given in GeV units, and all points are chosen so as to be consistent with mass and B-physics constraints. Point 1 depicts a solution with exact degeneracy between stop and top quarks. Point 2 and point 3 display the heaviest gluino and the heaviest Higgs boson solutions, respectively. Point 4 represents a solution that is consistent with WMAP bounds on the relic abundance of the neutralino LSP.

\begin{tabular}{|c|c|c|c|c|}
\hline & Point 1 & Point 2 & Point 3 & Point 4 \\
\hline$m_{0}$ & 5051 & 5641 & 6267 & 5796 \\
\hline$m_{10}$ & 45.3 & 21.9 & 190 & 76.6 \\
\hline$M_{1 / 2}$ & 359.4 & 393.1 & 341.7 & 335 \\
\hline $\tan \beta$ & 18.0 & 17.9 & 20.6 & 19.4 \\
\hline$A_{0} / m_{0}$ & -2.64 & -2.64 & -2.67 & -2.66 \\
\hline$m_{t}$ & 173.3 & 173.3 & 173.3 & 173.3 \\
\hline$\mu$ & 6168 & 6887 & 7612 & 7050 \\
\hline$\Delta a_{\mu}$ & $0.77 \times 10^{-11}$ & $0.60 \times 10^{-11}$ & $0.49 \times 10^{-11}$ & $0.56 \times 10^{-11}$ \\
\hline$m_{h}{ }^{m}$ & 123.4 & 123.8 & 125.3 & 125.1 \\
\hline$m_{H}$ & 5591 & 6258 & 6647 & 6266 \\
\hline$m_{A}$ & 5556 & 6218 & 6604 & 6225 \\
\hline$m_{H^{ \pm}}$ & 5593 & 6260 & 6648 & 6267 \\
\hline$m_{\tilde{\chi}_{1}^{0}}$ & $170.4,340.6$ & $187.4,374.1$ & $166.9,335.8$ & $162.1,325.9$ \\
\hline$m_{\tilde{\tilde{\alpha}}}^{u^{1,2}}$ & 6159,6159 & 6877,6878 & 7600,7600 & 7040,7040 \\
\hline$m_{\tilde{\gamma}_{1,4}^{ \pm}}^{n_{1}}$ & $\mathbf{3 4 3}, 6165$ & 376.7, 6884 & 338.1, 7606 & 328.1, 7046 \\
\hline$m_{\tilde{g}}^{\chi_{1,2}}$ & 1047 & 1189 & 1054 & 1020 \\
\hline$m_{\tilde{u}_{L R}}^{g}$ & 5071,5083 & 5662,5676 & 6269,6286 & 5801,5816 \\
\hline$m_{t_{1,2}}^{u_{1}}$ & $173.3,3313$ & $189.6,3702$ & $198.5,3985$ & $200.2,3735$ \\
\hline$m_{\tilde{d}_{1,2}}$ & 5072,5083 & 5663,5676 & 6270,6286 & 5802,5817 \\
\hline$m_{\tilde{L}, R}$ & 3346,4694 & 3743,5250 & 4026,5659 & 3773,5300 \\
\hline$m_{\tilde{\nu}_{e, ~}}$ & 5059 & 5650 & 6275 & 5804 \\
\hline$m_{\tilde{\nu}_{-}, \mu}$ & 4842 & 5412 & 5929 & 5517 \\
\hline$m_{\tilde{e}_{L R}}$ & 5053,5048 & 5643,5637 & 6267,6262 & 5796,5792 \\
\hline$m_{\tilde{\tau}_{1,2}}$ & 4621,4847 & 5170,5417 & 5581,5937 & 5229,5524 \\
\hline$\sigma_{\mathrm{SI}}(\mathrm{pb})$ & $0.32 \times 10^{-11}$ & $0.20 \times 10^{-11}$ & $0.14 \times 10^{-11}$ & $0.11 \times 10^{-11}$ \\
\hline$\sigma_{\mathrm{SD}}(\mathrm{pb})$ & $0.61 \times 10^{-11}$ & $0.41 \times 10^{-11}$ & $0.27 \times 10^{-11}$ & $0.37 \times 10^{-11}$ \\
\hline$\Omega h^{2}$ & $0.14 \times 10^{-3}$ & $0.18 \times 10^{-3}$ & 0.07 & 0.11 \\
\hline$\Delta_{\mathrm{EW}}$ & 9206 & 11478 & 14044 & 12037 \\
\hline$\Delta_{\mathrm{BG}}$ & 9207 & 11478 & 14052 & 12039 \\
\hline
\end{tabular}

Point 1 depicts a solution with exact degeneracy between the stop and top quarks. Point 2 and point 3 display the heaviest gluino and the heaviest Higgs boson solutions, respectively. Point 4 represents a solution that is consistent with the WMAP bound on the relic abundance of the neutralino LSP. The main lesson from this section is that nonuniversality in Higgs mass parameters typically reduces fine-tuning from the $10^{4}$ to $10^{3}$ level. This is good but certainly insufficient for having a sensible parameter domain for stop-top degeneracy.

\section{GRAVITINO LSP}

In Secs. III and IV, we have considered and revealed our results for solutions with $\left|m_{\tilde{t}}-m_{t}\right| \leq 30 \mathrm{GeV}$ in the CMSSM and NUHM1. In scanning the parameter spaces of these models, we have accepted only the solutions that are compatible with the neutralino LSP and found that the mass difference between the NLSP stop and LSP neutralino up to $20 \%$ is excluded by the WMAP bound. However, there are some other possible dark matter candidates in the
MSSM such as the sneutrino and gravitino. In the case of the left-handed sneutrino LSP, the sneutrinos lighter than $25 \mathrm{GeV}$ have been excluded by LEP searches for $\mathrm{Z}$ decays, while those heavier than $25 \mathrm{GeV}$ would have already been observed in direct detection experiments [52]. Then, one may consider the gravitino LSP case as an alternative to the neutralino LSP cases. Although its interactions are too weak to be detected at the LHC, the gravitino LSP is strongly constrained by big bang nucleosynthesis (BBN) [53], observed abundances of primordial light elements such as $\mathrm{D}, \mathrm{He}$, and Li [54], and by the LHC and WMAP constraints. Especially, BBN stringently constrains the gravitino LSP. Also, since gravitino relic abundance receives contributions from decays of the NLSP, the properties of the NLSP become important and constrained by the cosmological observations. Each kind of NLSP has its own phenomenology, and many possibilities have been studied such as the neutralino, stau, sneutrino, and stop [55,56].

The gravitino mass is proportional to the SUSY breaking scale 


$$
m_{3 / 2} \sim \frac{\langle F\rangle}{\sqrt{3} M_{\mathrm{Pl}}},
$$

where $m_{3 / 2}$ is the gravitino mass, $F$ is the SUSY breaking scale squared in the hidden sector, and $M_{\mathrm{P}}$ is the Planck scale. Here, $\langle F\rangle$ depends on the types of the messengers that mediate SUSY breaking from the hidden sector to the visible one. In the gauge mediated SUSY breaking scenario, $\langle F\rangle \sim 10^{8}-10^{19}$; hence, the gravitino mass is in the range of $0.1 \mathrm{eV}-10 \mathrm{GeV}$ [57], while in gravity mediated supersymmetry breaking, $\langle F\rangle \sim 10^{21}-10^{22} \mathrm{GeV}^{2}$, and hence $m_{3 / 2} \sim 100-1000 \mathrm{GeV}$ [58]. Note that it is also possible to vary $m_{3 / 2}$ as a free parameter of a model by keeping the gravitino as the LSP [56].

In the supersymmetric models considered in this paper, SUSY is broken via gravity mediation, and gravitino is necessarily in the spectrum. In this section, we consider the CMSSM in the framework of gravity-mediated SUSY breaking with the gravitino LSP for which the mass is assumed to be $\lesssim 100 \mathrm{GeV}$. The presence of the gravitino does not affect significantly the remaining sparticle spectrum. The fact that the gravitino itself is the LSP relaxes strong restrictions on the CMSSM parameter space. As we adopted in the previous sections, we search for the regions with $m_{\tilde{t}} \simeq m_{t}$. It also leads to the NLSP stop with the gravitino LSP, and hence it is constrained by the cosmological constraints as well as the gravitino. Indeed, BBN constrains the lifetime and decays of the NLSP [53], and the bound on the stop is much stronger than other possibilities. Even for $m_{3 / 2} \sim 10 \mathrm{GeV}$, the lightest stop should be $\sim 1 \mathrm{TeV}$, and it is consistent with the results of Ref. [56] that did not find any solution for the NLSP stop in the CMSSM. To avoid the stringent bounds, we can assume the presence of a slight R-parity violation (RPV) that is consistent with the stops lighter than $200 \mathrm{GeV}$ when $\epsilon \gtrsim 10^{-7}$ [59], where $\epsilon$ measures the RPV. In this case, gravitino decays are suppressed by the small R-parity breaking parameter as well as the Planck mass, and hence it forms a viable dark matter even in the case of RPV.

With these assumptions, our results in Fig. 7 show that solutions with $m_{\tilde{t}} \sim m_{t}$ can be realized already in the CMSSM. All points are consistent with REWSB. Green points satisfy mass bounds and B-physics mentioned in Sec. II. Red points from a subset of green, and they satisfy $\Delta m_{\tilde{t} t} \leq 30 \mathrm{GeV}$, where $\Delta m_{\tilde{t} t} \equiv m_{\tilde{t}_{1}}-m_{t}$. The dashed line corresponds to $m_{\tilde{t}_{1}}=m_{t}=173.3 \mathrm{GeV}$. The plots show that the region that yields the degenerate top and stop is much wider than that found in the CMSSM with the neutralino LSP. As shown in the $M_{1 / 2}-m_{0}$ plane, degeneracy can be realized for $4 \lesssim m_{0} \lesssim 6 \mathrm{TeV}$, while $M_{1 / 2}$ is lifted up to $800 \mathrm{GeV}$ or so. Also, $\tan \beta$ is found to lie in a wide range from 10 to 50 , as shown in the $M_{1 / 2}-\tan \beta$ plane. The $M_{1 / 2}-A_{0} / m_{0}$ plane indicates that one needs a large mixing between left- and right-handed stops as in the CMSSM with the neutralino LSP, but in the gravitino LSP case, the mixing has opposite sign. With this mixing, the heavier stop is found to weigh $\gtrsim 3 \mathrm{TeV}$, as expected.
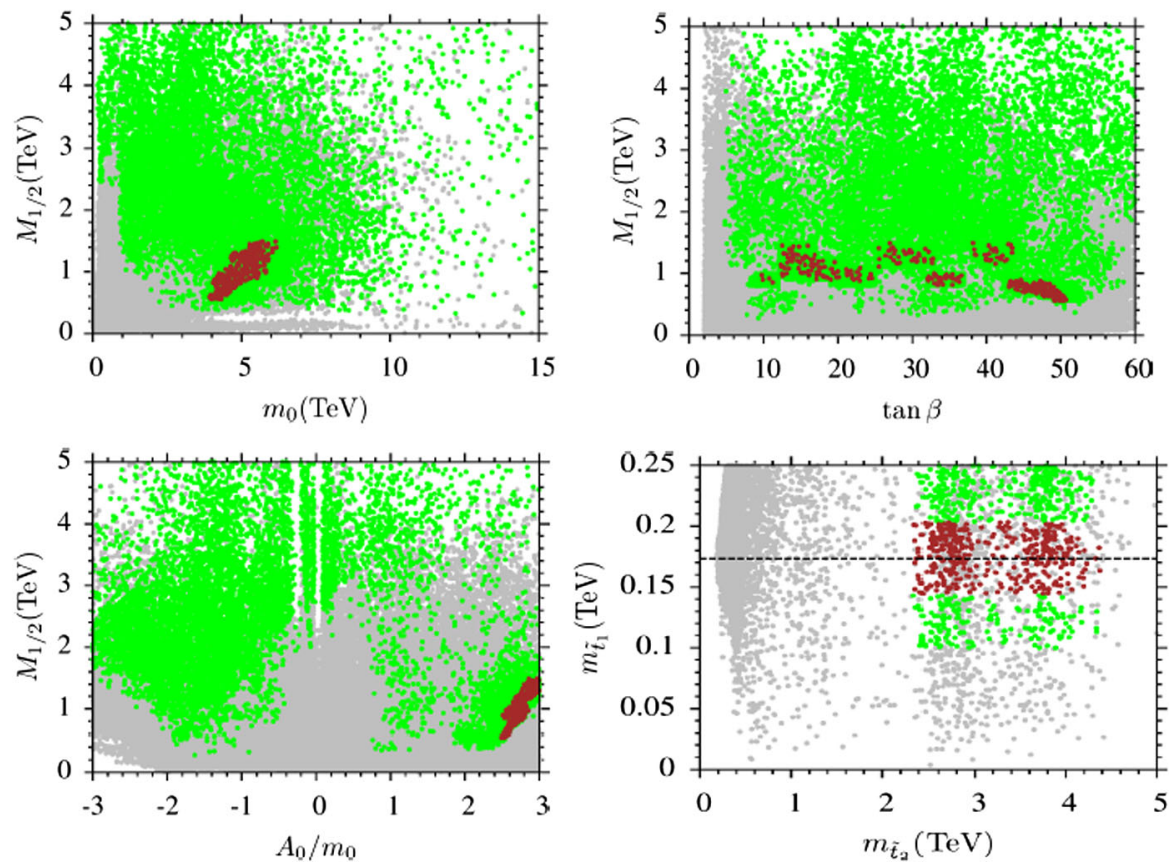

FIG. 7 (color online). Plots in the $M_{1 / 2}-m_{0}, M_{1 / 2}-\tan \beta, M_{1 / 2}-A_{0} / m_{0}$, and $m_{\tilde{t}_{1}}-m_{\tilde{t}_{2}}$ planes for the CMSSM with the gravitino LSP. All points are consistent with REWSB. Green points satisfy mass bounds and B-physics mentioned in Sec. II. Red points form a subset of the green and satisfy $\Delta m_{\tilde{t} t} \leq 30 \mathrm{GeV}$, where $\Delta m_{\tilde{t} t} \equiv m_{\tilde{t}_{1}}-m_{t}$. The dashed line corresponds to the top quark mass $m_{t}=173.3 \mathrm{GeV}$. 

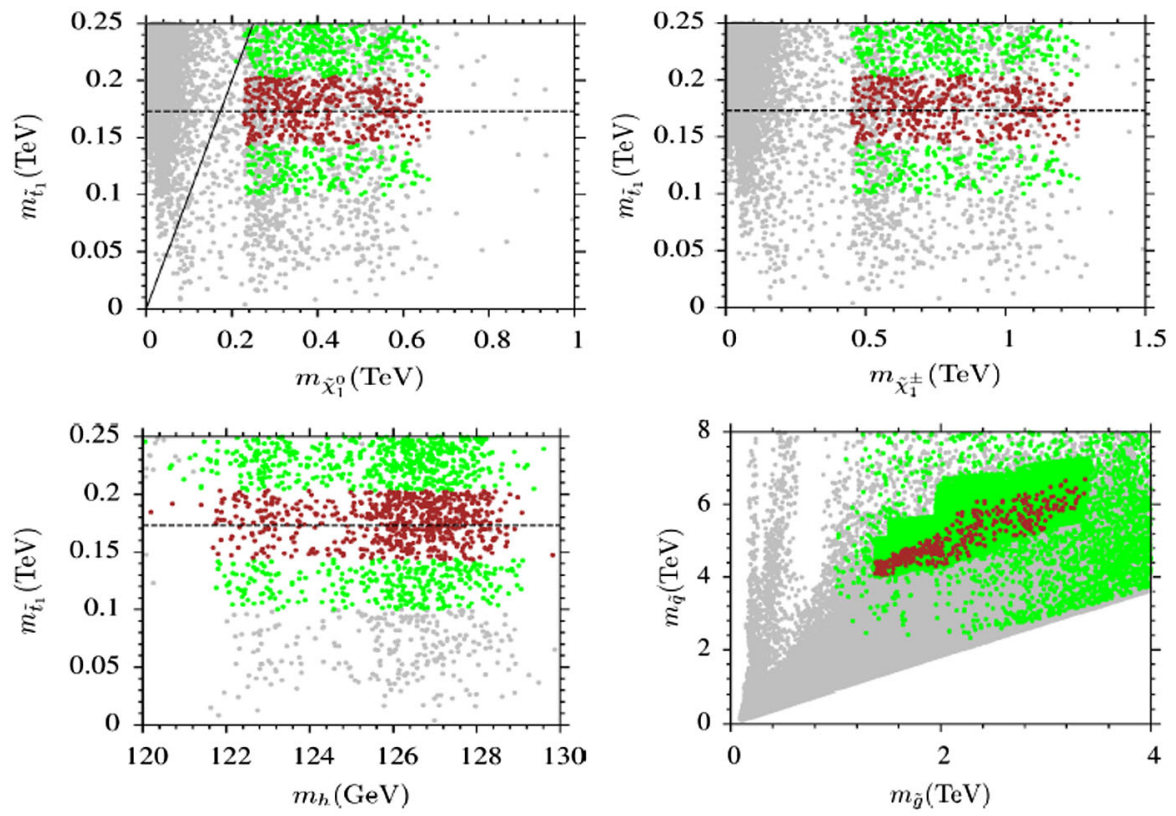

FIG. 8 (color online). Plots in the $m_{\tilde{t}_{1}}-m_{\tilde{\chi}_{1}^{0}}, m_{\tilde{t}_{1}}-m_{\tilde{\chi}_{1}^{ \pm}}, m_{\tilde{t}_{1}}-m_{h}$, and $m_{\tilde{q}}-m_{\tilde{g}}$ planes for the CMSSM with the gravitino LSP. The color coding is the same as Fig. 7 . The solid line in $m_{\tilde{t}_{1}}-m_{\tilde{\chi}_{1}^{0}}$ indicates the region where $m_{\tilde{t}_{1}}=m_{\tilde{\chi}_{1}^{0}}$.

The spectra obtained from our data are represented in the $m_{\tilde{t}_{1}}-m_{\tilde{\chi}_{1}^{0}}, m_{\tilde{t}_{1}}-m_{\tilde{\chi}_{1}^{ \pm}}, m_{\tilde{t}_{1}}-m_{h}$, and $m_{\tilde{q}}-m_{\tilde{g}}$ planes of Fig. 8. The color coding is the same as in Fig. 7. The solid line in $m_{\tilde{t}_{1}}-m_{\tilde{\chi}_{1}^{0}}$ indicates the region in which $m_{\tilde{t}_{1}}=m_{\tilde{\chi}_{1}^{0}}$. The neutralino is found to be heavier $(\sim 200-600 \mathrm{GeV})$ than the stop all over the region, and, as stated above, this region is excluded, since a charged sparticle becomes a LSP unless the gravitino is the LSP. Once we allow the stop to be lighter than the neutralino, the chargino is also found to be heavier than those in the CMSSM with the neutralino LSP.
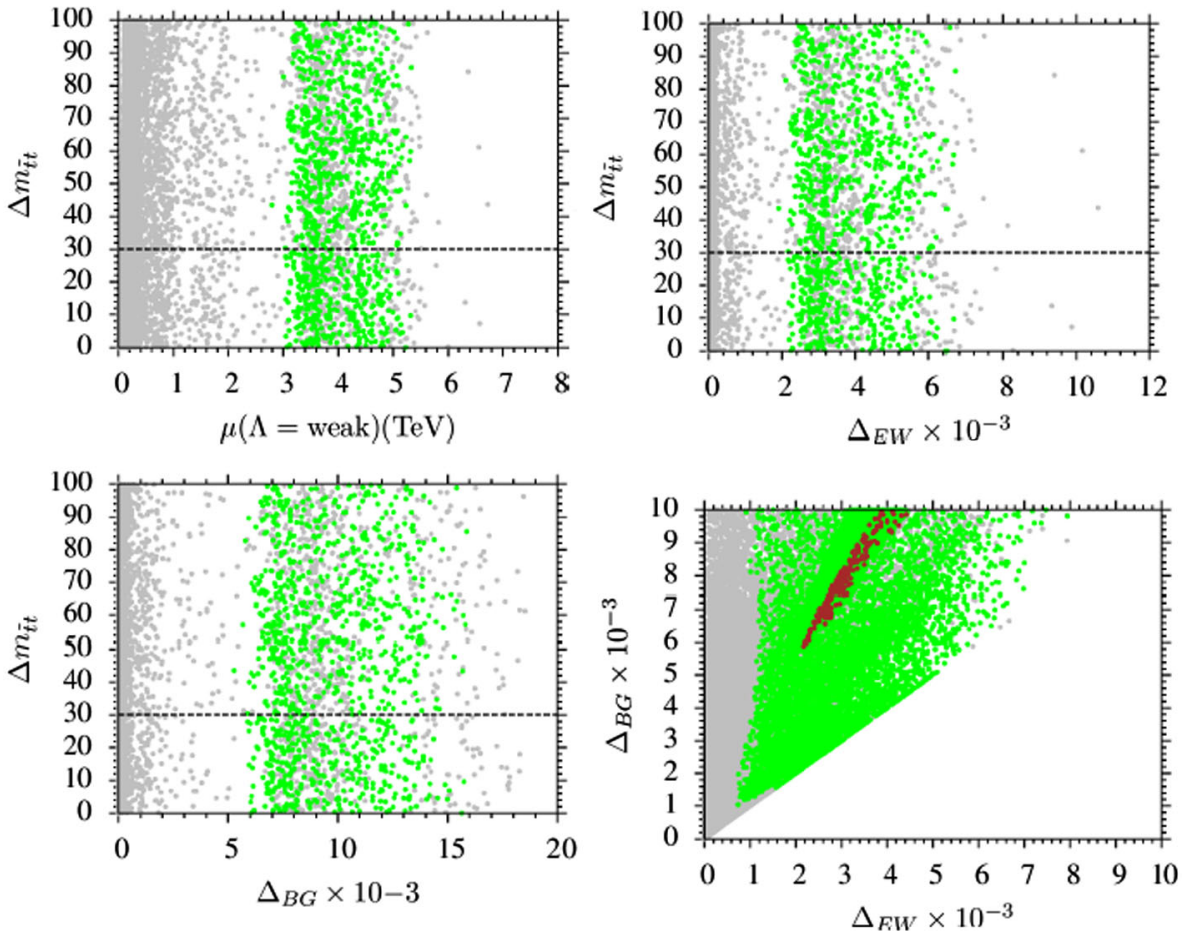

FIG. 9 (color online). Fine-tuning plots in the $\Delta m_{\tilde{t} t}-\mu, \Delta m_{\tilde{t} t}-\Delta_{\mathrm{EW}}, \Delta m_{\tilde{t} t}-\Delta_{\mathrm{BG}}$, and $\Delta_{\mathrm{BG}}-\Delta_{\mathrm{EW}}$ planes for the CMSSM with the gravitino LSP. The color coding is the same as Fig. 3. 
The $m_{\tilde{t}_{1}}-m_{\tilde{\chi}_{1}^{0}}$ plane shows the range for the lightest chargino mass as 500-1200 GeV. Similarly, we have a much wider mass range for the Higgs boson mass as $\sim 122-130 \mathrm{GeV}$. The squarks of the first two generations are found to be lighter $(\sim 4-7 \mathrm{TeV})$, while the gluinos are heavy $(\sim 1.5-3.5 \mathrm{TeV})$, and they can be tested in the LHC14 run.

Figure 9 displays the result for the fine-tuning in the $\Delta m_{\tilde{t} t}-\mu, \quad \Delta m_{\tilde{t} t}-\Delta_{\mathrm{EW}}, \quad \Delta m_{\tilde{t} t}-\Delta_{\mathrm{BG}}$, and $\Delta_{\mathrm{BG}}-\Delta_{\mathrm{EW}}$ planes. The color coding is the same as Fig. 3. The CMSSM with the gravitino LSP has slightly better results compared to those obtained for the CMSSM with the neutralino LSP; $\quad \mu(\Lambda=$ weak $) \sim 3-5 \mathrm{TeV}, \quad \Delta_{\mathrm{EW}} \sim$ 2000-6000 (0.05\%-0.017\%), and $\Delta_{\mathrm{BG}} \sim 5000-15000$ $(0.02 \%-0.007 \%)$.

Finally, we display five benchmark points that exemplify our results for the CMSSM with the gravitino LSP in Table III. Masses are given in $\mathrm{GeV}$ units. All points are chosen so as to be consistent with the LHC bounds. Point 1 depicts a solution with exact degeneracy between stop and top quarks, and points 2 and 3 display solutions with the Higgs boson mass measured by ATLAS and CMS. Points 4 and 5 display solutions with different $\tan \beta$. The lesson from this section is that the gravitino does not add much to the NUHM1 in reducing the fine-tuning.

\section{CMSSM WITH $\mu<0$ AND NONUNIVERSAL GAUGINO MASSES}

So far, we have considered the stop-top degeneracy region in the CMSSM and NUHM1 for both the neutralino LSP and gravitino LSP cases. The results show that it is possible to find such light stops for which the pair production stays in the error bar in the calculation of the top quark pair production. While such regions are realized consistently with the experimental constraints, our results show that the models need to be highly fine-tuned. The best results for fine-tuning are obtained for the case with the gravitino LSP $\left(\Delta_{\mathrm{EW}} \sim 2000\right)$, while it is worse for the cases with the neutralino LSP in both the $\operatorname{CMSSM}\left(\Delta_{\mathrm{EW}} \sim 9000\right)$ and NUHM1 $\left(\Delta_{\mathrm{EW}} \sim 8000\right)$. If one excludes solutions requiring $\Delta_{\text {EW }} \lesssim 1000$, then a stop-top degeneracy region in all the models considered in this paper disappears by the fine-tuning constraint.

In this section, we discuss nonuniversality in gaugino masses. In general, fine-tuning indicates that there is a missing mechanism in the model under concern, and its amount can be interpreted as the effectiveness of the missing mechanism in the considered regions. There are exclusive studies on fine-tuning in supersymmetric models (for an incomplete list, see Ref. [26]). The results from

TABLE III. Benchmark points for the CMSSM with the gravitino LSP. Masses are given in GeV units. All points are chosen si as to be consistent with the LHC bounds. Point 1 depicts a solution with exact degeneracy between stop and top quarks, and points 2 and 3 display solutions with the Higgs boson mass measured by ATLAS and CMS. Points 4 and 5 display solutions with different tan $\beta$.

\begin{tabular}{|c|c|c|c|c|c|}
\hline & Point 1 & Point 2 & Point 3 & Point 4 & Point 5 \\
\hline$m_{0}$ & 6170 & 5753 & 5733 & 5244 & 5376 \\
\hline$M_{1 / 2}$ & 1396 & 1198 & 1103 & 1298 & 1363 \\
\hline $\tan \beta$ & 27.2 & 18.0 & 16.6 & 38.6 & 41.0 \\
\hline$A_{0} / m_{0}$ & 2.80 & 2.71 & 2.65 & 2.92 & 2.95 \\
\hline$m_{t}$ & 173.3 & 173.3 & 173.3 & 173.3 & 173.3 \\
\hline$\mu$ & 5091 & 4678 & 4574 & 4455 & 4596 \\
\hline$\Delta a_{\mu}$ & $0.13 \times 10^{-11}$ & $0.94 \times 10^{-11}$ & $0.87 \times 10^{-11}$ & $0.25 \times 10^{-10}$ & $0.25 \times 10^{-10}$ \\
\hline$m_{h}{ }^{\mu}$ & 126.9 & 125.6 & 125.6 & 125.9 & 126.1 \\
\hline$m_{H}$ & 6980 & 7019 & 7012 & 5190 & 5125 \\
\hline$m_{A}$ & 6935 & 6973 & 6966 & 5156 & 5092 \\
\hline$m_{H^{ \pm}}$ & 6981 & 7019 & 7012 & 5190 & 5126 \\
\hline$m_{\tilde{\chi}^{0}}$ & $615.8,1166$ & $526.8,1002$ & $483.8,922.1$ & $567.2,1076$ & $596.1,1130$ \\
\hline$m_{\tilde{\chi}_{3,4}^{0}}^{\lambda_{1,2}}$ & 5127,5128 & 4717,4718 & 4619,4620 & 4477,4478 & 4613,4614 \\
\hline$m_{\tilde{\chi}_{12}^{ \pm}}^{\lambda_{3,4}}$ & 1180, 5159 & 1014, 4749 & $\mathbf{9 3 4 . 3}, 4652$ & $\mathbf{1 0 8 8}, 4502$ & 1142, 4637 \\
\hline$m_{\tilde{g}}^{\chi_{1,2}}$ & 3190 & 2784 & 2590 & 2960 & 3091 \\
\hline$m_{\tilde{u}_{L, R}}^{9}$ & 6656,6633 & 6138,6123 & 6058,6048 & 5750,5724 & 5918,5890 \\
\hline$m_{\tilde{t}_{1,2}}^{u_{L}}$ & $173.3,4328$ & $157.9,4148$ & $183.6,4111$ & $169.4,3569$ & $172.9,3645$ \\
\hline$m_{\tilde{d}_{L R}}$ & 6656,6633 & 6138,6121 & 6058,6046 & 5751,5724 & 5919,5886 \\
\hline$m_{\tilde{b}_{12}}^{u_{L, R}}$ & 4370,6020 & 4187,5797 & 4150,5761 & 3599,4921 & 3675,5009 \\
\hline$m_{\tilde{\nu}_{e, \mu}}^{b_{1,2}}$ & 6234 & 5803 & 5776 & 5309 & 5446 \\
\hline$m_{\tilde{\nu}_{\tau}}$ & 5597 & 5541 & 5758 & 4202 & 4156 \\
\hline$m_{\tilde{e}_{L, R}}$ & 6225,6186 & 5794,5765 & 5767,5742 & 5302,5262 & 5439,5396 \\
\hline$m_{\tilde{\tau}_{1,2}}$ & 4884,5624 & 5249,5550 & 5316,5564 & 2705,4238 & 2249,3645 \\
\hline$\Delta_{\mathrm{EW}}$ & 6463 & 5398 & 5163 & 4954 & 5258 \\
\hline$\Delta_{\mathrm{BG}}$ & 15636 & 13389 & 13102 & 11576 & 12216 \\
\hline
\end{tabular}



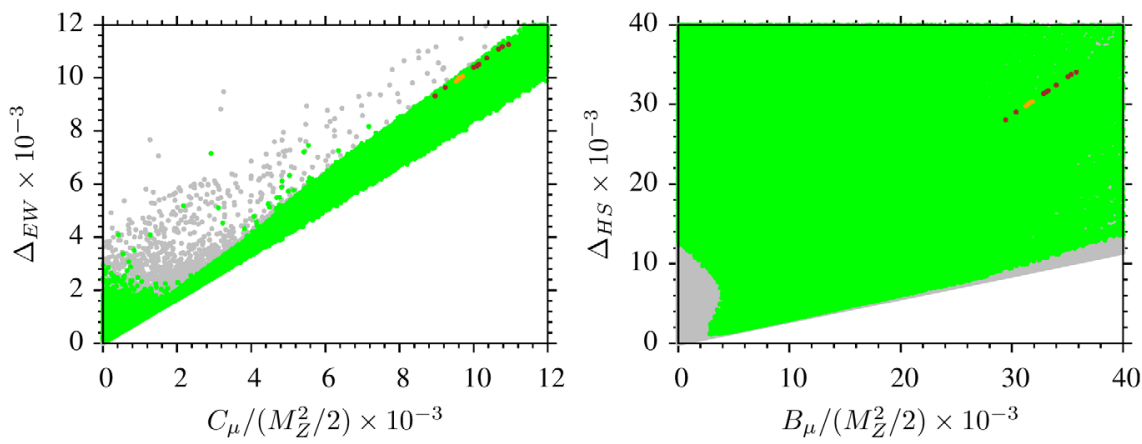

FIG. 10 (color online). Plots in the $\Delta_{\mathrm{EW}}-C_{\mu}$ and $\Delta_{\mathrm{HS}}-B_{\mu}$ planes. $C_{\mu}$ and $B_{\mu}$ are defined in Eqs. (4) and (6). All points are consistent with REWSB and the neutralino LSP. Green points satisfy mass bounds and constraints from B physics, and red points are a subset of green and satisfy the $\Delta m_{\tilde{t} t} \leq 50 \mathrm{GeV}$. Orange points are a subset of red, and they yield the relic density of the neutralino LSP less than 1 .

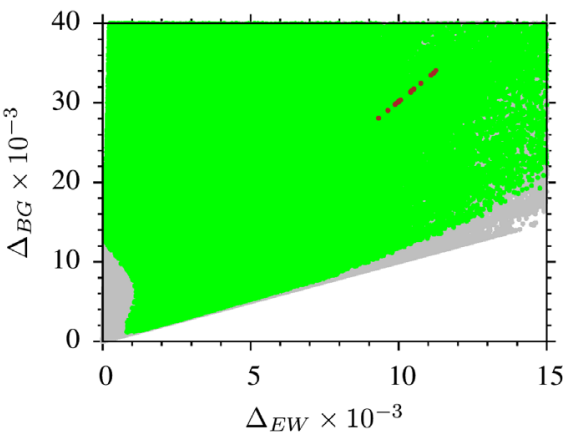

(a) $\mu>0$

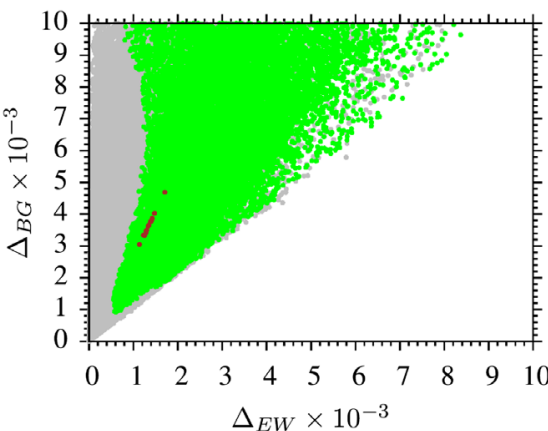

(b) $\mu<0$

FIG. 11 (color online). Plots in the $\Delta_{\mathrm{BG}}-\Delta_{\mathrm{HS}}$ panels for the CMSSM. The color coding is the same as Fig. 10. The left panel is for $\mu>0$, and the right panel is for $\mu<0$.

those studies show that the fine-tuning constraint brings a lower bound on the stop mass around $500 \mathrm{GeV}$, even in extensions of the MSSM. As discussed in Secs. III, IV, and $\mathrm{V}$, the stop-top degeneracy region requires a large mixing between stops. The connection between the finetuning and the large mixing can be established by considering the $\mu$ term. The mixing between the stops is proportional to $A_{t}-\mu \cot \beta$, and hence the $\mu$ term balances the contributions from large $A_{t}$ in order to adjust the stop mass such that it turns out to be nearly degenerate with the top quark. Besides, the $\mu$ term also determines the amount of fine-tuning as shown in plots in the $\Delta_{\mathrm{EW}}-C_{\mu}$ and $\Delta_{\mathrm{HS}}-$ $B_{\mu}$ planes of Fig. 10. All points in these plots are consistent with REWSB and the neutralino LSP. Green points satisfy mass bounds and constraints from B physics, and red points are a subset of green and satisfy the $\Delta m_{\tilde{t} t} \leq 50 \mathrm{GeV}$. Orange points are a subset of red, and they yield the relic density of the neutralino LSP less than 1. The linear correlations in Fig. 10 mean that $\Delta_{\mathrm{EW}}$ is determined by $C_{\mu}$ and $\Delta_{\mathrm{BG}}$ by $B_{\mu}$.

The minimization of the Higgs potential allows both negative and positive signs for $\mu$, and hence one can consider the case with negative $\mu$, which reverses the effect of $\mu$ in the mixing of two stop quarks. The effect of the sign of $\mu$ on the fine-tuning is shown for the CMSSM in the
$\Delta_{\mathrm{BG}}-\Delta_{\mathrm{HS}}$ panels of Fig. 11 . The negative $\mu$ is seen to reduce fine-tuning by an order of magnitude.

Even though a significant improvement is realized in the case with $\mu<0\left(\Delta_{\mathrm{EW}} \sim 2000\right)$, it is still excluded by the constraint $\Delta_{\mathrm{EW}} \lesssim 1000$. Can we further lower fine-tuning? As we remember from nonuniversal Higgs masses, this can happen only if we increase the degrees of freedom. Namely, we must further deviate from CMSSM conditions. To this end, we explore the effects of nonunivesal gaugino masses. We study the CMSSM with $\mu<0$ and nonuniversal gauginos and give the required fine-tuning for the stoptop degeneracy region by scanning the model parameters in the following ranges:

$$
\begin{array}{r}
0 \leq m_{0} \leq 20 \mathrm{TeV} \\
0 \leq M_{1} \leq 5 \mathrm{TeV} \\
0 \leq M_{2} \leq 5 \mathrm{TeV} \\
0 \leq M_{3} \leq 5 \mathrm{TeV} \\
-3 \leq A_{0} / m_{0} \leq 3 \\
2 \leq \tan \beta \leq 60 \\
\mu<0,
\end{array}
$$



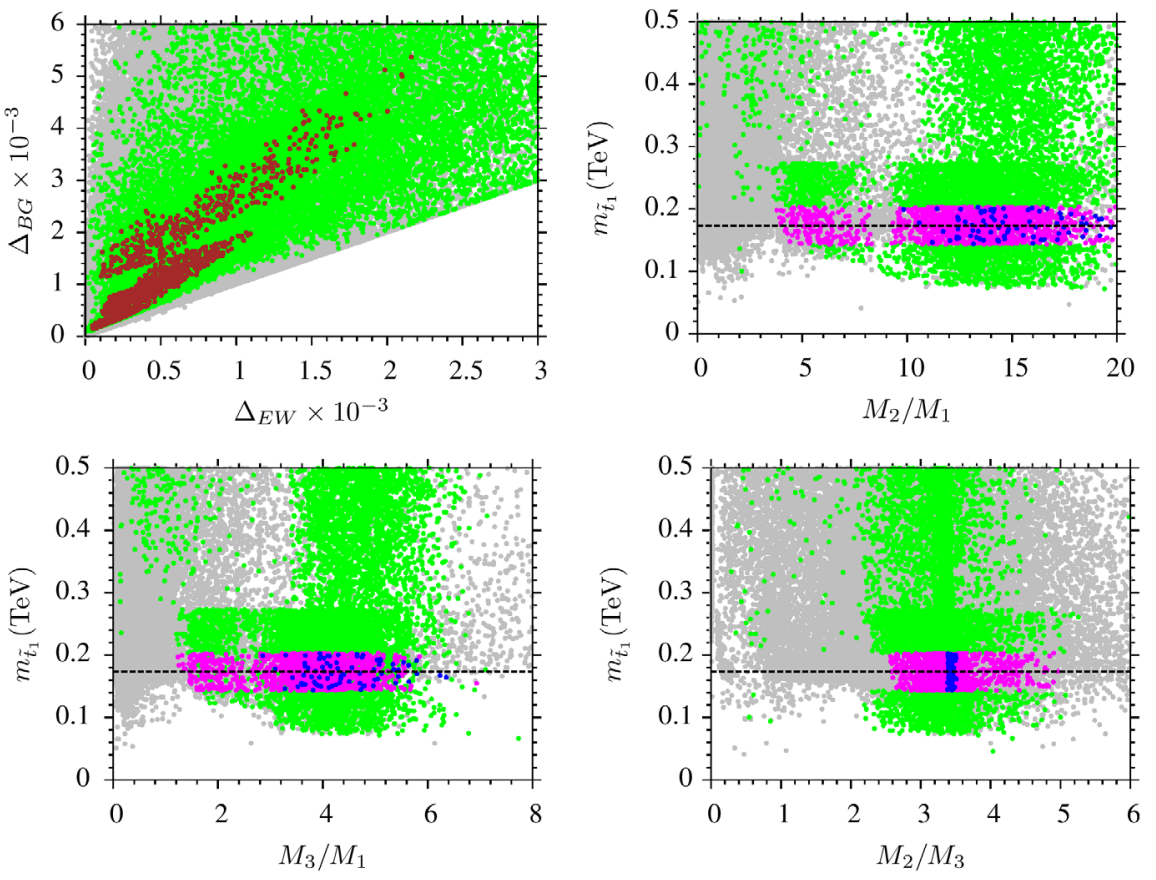

FIG. 12 (color online). Plots in the $\Delta_{\mathrm{BG}}-\Delta_{\mathrm{EW}}, m_{\tilde{t}_{1}}-M_{2} / M_{1}, m_{\tilde{t}_{1}}-M_{2} / M_{3}$, and $m_{\tilde{t}_{1}}-M_{3} / M_{1}$ planes. All points are consistent with REWSB and the neutralino LSP. Green points satisfy the mass bounds and constraints from B physics. Red points are a subset of green and satisfy $\Delta m_{\tilde{t} t} \leq 30 \mathrm{GeV}$. Magenta points form a subset of orange and represent the regions with $\Delta_{\mathrm{EW}} \leq 500$. Similarly, the blue points form a subset of magenta, and they satisfy $\Delta_{\mathrm{EW}} \leq 100$.

where the sign of $\mu$ directly influences the stop left-right mixing and hence the mass splitting between the two mass eigenstates.

Figure 12 represents plots in the $\Delta_{\mathrm{BG}}-\Delta_{\mathrm{EW}}$, $m_{\tilde{t}_{1}}-M_{2} / M_{1}, m_{\tilde{t}_{1}}-M_{2} / M_{3}, m_{\tilde{t}_{1}}-M_{3} / M_{1}$ planes. All points are consistent with REWSB and the neutralino LSP. Green points satisfy the mass bounds and constraints from B physics. Orange points are a subset of green and satisfy $\Delta m_{\tilde{t} t} \leq 30 \mathrm{GeV}$. Magenta points form a subset of orange and represent the regions with $\Delta_{\mathrm{EW}} \leq 500$. Similarly, the blue points form a subset of magenta, and they satisfy $\Delta_{\mathrm{EW}} \leq 100$. It can be seen that plenty of solutions for $\Delta_{\mathrm{EW}} \leq 500$ can be realized in a model with nonuniversal gaugino masses at $M_{\mathrm{GUT}}$ and $\mu<0$. From the plots in the $m_{\tilde{t}_{1}}-M_{2} / M_{1}$ and $m_{\tilde{t}_{1}}-M_{3} / M_{1}$ planes, we find the ratios for the gaugino masses as $M_{2} / M_{1} \gtrsim 5$ and $M_{3} / M_{1} \gtrsim 2$. As can be seen from $m_{\tilde{t}_{1}}-M_{2} / M_{3}$, the ratio of $M_{2}$ to $M_{3}$ is found to be in a range $3 \lesssim M_{2} / M_{3} \lesssim 5$ for $\Delta_{E W} \leq 500$, while $M_{2} / M_{3} \approx 3.5$ if one applies the condition $\Delta_{\text {EW }} \leq 100$ strictly. It is clear that having an $S U(2)$ gaugino so heavy compared to $S U(3)$ and $U(1)_{Y}$ gauginos blatantly violates the CMSSM conditions at a high scale. In conclusion, compared to the CMSSM analyzed in Sec. III, its nonuniversal Higgs mass extension in Sec. IV, and its supergravity structure in Sec. V, only the CMSSM $\mu<0$ extended by nonuniversal gaugino masses proves a viable reduction in fine-tuning. The fine-tuning cost of realizing stop-top degeneracy falls from $\mathcal{O}\left(10^{4}\right)$ in the CMSSM down to $\mathcal{O}(100)$ with nonuniversal gaugino masses and $\mu<0$.

\section{CONCLUSION}

In this work, we have searched for parameter regions that yield a light stop that is nearly degenerate with the top quark. Being the region not yet examined at the LHC, this narrow stripe is the place a light stop can be hidden. Indeed, the LHC constraints for colored sparticles are severe and follow from nonobservation of any of these sparticles. However, the cross section of stop pair production is so small that it is less than the experimental error bar in the measurement of the top pair production. Namely, light stops can hide in strong top quark background, and it is difficult to distinguish them from the top quark signals (unless one performs precise spin measurements).

We performed in this work a detailed search to determine if the CMSSM paramater space can accommodate a light stop nearly degenerate in mass with a top quark. We explored degeneracy in a narrow band less than $50 \mathrm{GeV}$. In Sec. III, we considered the CMSSM parameter space with $m_{0} \sim 9 \mathrm{TeV}, M_{1 / 2} \sim 300 \mathrm{GeV}, A_{0} / m_{0} \sim-2.2$, and $\tan \beta \sim$ 34 while the squarks of the first two generations were kept heavy $(\sim 9 \mathrm{TeV})$ in agreement with the LHC constraints. The gluinos were found to be slightly above $1 \mathrm{TeV}$. On the other hand, the WMAP bound on the relic abundance of the neutralino LSP excluded the regions with mass difference between the NLSP stop and LSP neutralino up to $20 \%$ that make the degeneracy between stop and top worse. As we depicted in Sec. III, the stop-on-top scenario was realized by a fine-tuning $\mathcal{O}\left(10^{4}\right)$ even when the stop-top mass 
splitting was as large as $50 \mathrm{GeV}$. This fine-tuning is huge, and it tells us that there is something missing in modelling light scalar tops in the framework of the CMSSM.

We then started looking for extensions of the CMSSM in which fine-tuning can be lowered. We prefered to study cases in which the CMSSM gauge group and particle spectrum were held. Namely, we did not study extended models like next-to-MSSM. We started our exploration with nonuniversality in the mass parameters of the two Higgs doublets. The NUHM1 model was analyzed in Sec. IV and found to yield stop on top with fine-tunings of $\mathcal{O}\left(10^{3}\right)$. The CMSSM with gravitino LSP, as analyzed in Sec. V, required a similar order of fine-tuning. The CMSSM with $\mu<0$ and nonuniveral gaugino masses, however, turned out to require much lower fine-tunings $\mathcal{O}(100)$. This price payed for this gain in fine-tuning is the nonuniversality in gaugino masses, where the $S U(2)$ gaugino is more massive than the $S U(3)$ gaugino.

To sum up, the light stop band allowed by the LHC data was consistently realized in known models of supersymmetry at the expense of severe fine-tunings. The reason for fine-tuning is that stops must be light enough to facilitate a natural Higgs boson yet heavy enough to facilitate a heavy Higgs boson. Among conservative extensions of the CMSSM, only the one with $\mu<0$ and nonuniversal gaugino masses gives the least fine-tunings $\mathcal{O}(100)$. Further studies on CMSSM extensions can reveal more islands in the parameter space in which the stop-on-top scenario is naturally realized.

\section{ACKNOWLEDGMENTS}

We would like to thank Qaisar Shafi and Liucheng Wang for fruitful discussions. We thank also Florian Staub and Ben O'Leary for useful comments concerning CCB minima. This work is supported in part by the TAEK CERN Project No. CERN-A5.H2.P1.01-21 (DAD), in which discussions with Koray Sevim are acknowledged. This work used the Extreme Science and Engineering Discovery Environment (XSEDE), which is supported by the National Science Foundation Grant No. OCI-1053575, and part of the computations have been carried out at ULAKBIM high performance center.
[1] G. Aad et al. (ATLAS Collaboration), Phys. Lett. B 716, 1 (2012).

[2] S. Chatrchyan et al. (CMS Collaboration), Phys. Lett. B 716, 30 (2012); J. High Energy Phys. 06 (2013) 081 .

[3] E. Gildener, Phys. Rev. D 14, 1667 (1976); Phys. Lett. 92B, 111 (1980); S. Weinberg, Phys. Lett. 82B, 387 (1979); L. Susskind, Phys. Rev. D 20, 2619 (1979); M. J. G. Veltman, Acta Phys. Pol. B 12, 437 (1981).

[4] G. Degrassi, S. Di Vita, J. Elias-Miro, J. R. Espinosa, G. F. Giudice, G. Isidori, and A. Strumia, J. High Energy Phys. 08 (2012) 098; F. Bezrukov, M. Y. Kalmykov, B. A. Kniehl, and M. Shaposhnikov, J. High Energy Phys. 10 (2012) 140; D. Buttazzo, G. Degrassi, P. P. Giardino, G. F. Giudice, F. Sala, A. Salvio, and A. Strumia, J. High Energy Phys. 12 (2013) 089; V. Branchina and E. Messina, Phys. Rev. Lett. 111, 241801 (2013); V. Branchina, E. Messina, and A. Platania, arXiv:1407.4112; V. Branchina, E. Messina, and M. Sher, arXiv:1408.5302.

[5] H. E. Haber, in Erice 1992, 23rd Eloisatron Workshop, Properties of SUSY Particles, LOCATION, 1992 (World Scientific, Erice, Italy, 1993), p. 321.

[6] ATLAS collaboration, Report No. ATLAS-CONF-2013047.

[7] L. Gouskos (CMS Collaboration), Proc. Sci., DIS 2013 (2013) 109.

[8] T. Lari (ATLAS Collaboration), Proc. Sci., EPS-HEP2013 (2014) 294.

[9] V. I. Martinez Outschoorn (CMS Collaboration), Eur. Phys. J. Web Conf. 60, 18003 (2013).
[10] ATLAS collaboration, Report No. ATLAS-CONF-2013068.

[11] D. COTE, Proc. Sci., EPS-HEP2013 (2014) 272.

[12] M. R. Buckley, T. Plehn, and M. J. Ramsey-Musolf, Phys. Rev. D 90, 014046 (2014).

[13] X.-Q. Li, Z.-G. Si, K. Wang, L. Wang, L. Zhang, and G. Zhu, Phys. Rev. D 89, 077703 (2014).

[14] K. Finelli (ATLAS Collaboration), Eur. Phys. J. Web Conf. 60, 16003 (2013); G. Aad et al. (ATLAS Collaboration), arXiv: 1406.5375.

[15] M. Carena, S. Gori, I. Low, N. R. Shah, and C. E. M. Wagner, J. High Energy Phys. 02 (2013) 114.

[16] M. Carena, S. Gori, N. R. Shah, and C. E. M. Wagner, J. High Energy Phys. 03 (2012) 014.

[17] U. Ellwanger and C. Hugonie, Phys. Lett. B 457, 299 (1999).

[18] J. E. Camargo-Molina, B. O'Leary, W. Porod, and F. Staub, J. High Energy Phys. 12 (2013) 103.

[19] J. E. Camargo-Molina, B. O'Leary, W. Porod, and F. Staub, Eur. Phys. J. C 73, 2588 (2013).

[20] R. Kitano and Y. Nomura, Phys. Lett. B 631, 58 (2005); Phys. Rev. D 73, 095004 (2006); C. Brust, A. Katz, S. Lawrence, and R. Sundrum, J. High Energy Phys. 03 (2012) 103; M. Papucci, J. T. Ruderman, and A. Weiler, J. High Energy Phys. 09 (2012) 035.

[21] C. Wymant, Phys. Rev. D 86, 115023 (2012); L. J. Hall, D. Pinner, and J. T. Ruderman, J. High Energy Phys. 04 (2012) 131; K. J. Bae, K. Choi, E. J. Chun, S. H. Im, C. B. Park, and C. S. Shin, J. High Energy Phys. 11 (2012) 118; K. J. Bae, T. H. Jung, and H. D. Kim, Phys. Rev. D 87, 015014 (2013); 
P. Athron, M. Binjonaid, and S. F. King, Phys. Rev. D 87, 115023 (2013); A. Katz, M. Reece, and A. Sajjad, arXiv:1406.1172.

[22] S. P. Martin, Perspectives on Supersymmetry II, edited by G. L. Kane (PUBLISHER, CITY OF PUBLICATION, DATE), p. 1 and references therein.

[23] H. Baer, V. Barger, P. Huang, D. Mickelson, A. Mustafayev, and X. Tata, Phys. Rev. D 87, 035017 (2013).

[24] R. Barbieri and G. F. Giudice, Nucl. Phys. B306, 63 (1988).

[25] H. Murayama, M. Olechowski, and S. Pokorski, Phys. Lett. B 371, 57 (1996).

[26] L. J. Hall, D. Pinner, and J. T. Ruderman, J. High Energy Phys. 04 (2012) 131; F. Brummer and W. Buchmuller, J. High Energy Phys. 05 (2012) 006; S. Caron, J. Laamanen, I. Niessen, and A. Strubig, J. High Energy Phys. 06 (2012) 008; Z. Kang, J. Li, and T. Li, J. High Energy Phys. 11 (2012) 024; T. Cheng, J. Li, T. Li, D. V. Nanopoulos, and C. Tong, Eur. Phys. J. C 73, 2322 (2013); M. Asano and T. Higaki, Phys. Rev. D 86, 035020 (2012); H. M. Lee, V. Sanz, and M. Trott, J. High Energy Phys. 05 (2012) 139; F. Brummer, S. Kraml, and S. Kulkarni, J. High Energy Phys. 08 (2012) 089; J. L. Feng and D. Sanford, Phys. Rev. D 86, 055015 (2012); K. Blum, R. T. D’Agnolo, and J. Fan, J. High Energy Phys. 01 (2013) 057; L. Randall and M. Reece, J. High Energy Phys. 08 (2013) 088; B. Kyae and J.-C. Park, Phys. Rev. D 87, 075021 (2013); H. Baer, V. Barger, P. Huang, A. Mustafayev, and X. Tata, Phys. Rev. Lett. 109, 161802 (2012); J. E. Younkin and S. P. Martin, Phys. Rev. D 85, 055028 (2012); S. Antusch, L. Calibbi, V. Maurer, M. Monaco, and M. Spinrath, J. High Energy Phys. 01 (2013) 187; H. Abe, T. Kobayashi, and Y. Omura, Phys. Rev. D 76, 015002 (2007); D. Horton and G. G. Ross, Nucl. Phys. B830, 221 (2010); I. Gogoladze, M. U. Rehman, and Q. Shafi, Phys. Rev. D 80, 105002 (2009); I. Gogoladze, F. Nasir, and Q. Shafi, Int. J. Mod. Phys. A 28, 1350046 (2013); J. High Energy Phys. 11 (2013) 173; E. Hardy, J. High Energy Phys. 10 (2013) 133; 03 (2014) 069; D. M. Ghilencea and G. G. Ross, Nucl. Phys. B868, 65 (2013); D. M. Ghilencea, H. M. Lee, and M. Park, J. High Energy Phys. 07 (2012) 046; S. Cassel, D. M. Ghilencea, and G. G. Ross, Nucl. Phys. B825, 203 (2010); D. M. Ghilencea, Nucl. Phys. B876, 16 (2013); P. Bechtle et al., J. High Energy Phys. 06 (2012) 098.

[27] B. C. Allanach, Comput. Phys. Commun. 143, 305 (2002).

[28] W. Porod, Comput. Phys. Commun. 153, 275 (2003).

[29] S. Heinemeyer, W. Hollik, and G. Weiglein, Comput. Phys. Commun. 124, 76 (2000).

[30] G. Isidori and P. Paradisi, Phys. Lett. B 639, 499 (2006); G. Isidori, F. Mescia, P. Paradisi, and D. Temes, Phys. Rev. D 75, 115019 (2007).

[31] G. Belanger, F. Boudjema, A. Pukhov, and A. Semenov, Comput. Phys. Commun. 149, 103 (2002); 185, 960 (2014).

[32] P. Gondolo, J. Edsjo, P. Ullio, L. Bergstrom, M. Schelke, and E. A. Baltz, J. Cosmol. Astropart. Phys. 07 (2004) 008.

[33] N. Nguyen, D. Horns, and T. Bringmann, arXiv:1202.1385.

[34] P. Bechtle, K. Desch, and P. Wienemann, Comput. Phys. Commun. 174, 47 (2006).

[35] O. Buchmueller et al., Eur. Phys. J. C 74, 2922 (2014).
[36] F. E. Paige, S. D. Protopopescu, H. Baer, and X. Tata, arXiv: hep-ph/0312045; for updates and changes in the current version see ISAJET-7.84.

[37] J. Hisano, H. Murayama, and T. Yanagida, Nucl. Phys. B402, 46 (1993); Y. Yamada, Z. Phys. C 60, 83 (1993); J. L. Chkareuli and I. G. Gogoladze, Phys. Rev. D 58, 055011 (1998).

[38] D. M. Pierce, J. A. Bagger, K. T. Matchev, and R.-j. Zhang, Nucl. Phys. B491, 3 (1997).

[39] ATLAS and CDF and CMS and D0 Collaborations, arXiv:1403.4427.

[40] I. Gogoladze, R. Khalid, S. Raza, and Q. Shafi, J. High Energy Phys. 04 (2014) 109.

[41] I. Gogoladze, R. Khalid, S. Raza, and Q. Shafi, J. High Energy Phys. 06 (2011) 117.

[42] G. Belanger, F. Boudjema, A. Pukhov, and R. K. Singh, J. High Energy Phys. 11 (2009) 026; H. Baer, S. Kraml, S. Sekmen, and H. Summy, J. High Energy Phys. 03 (2008) 056.

[43] L. E. Ibanez and G. G. Ross, Phys. Lett. 110B, 215 (1982); K. Inoue, A. Kakuto, H. Komatsu, and S. Takeshita, Prog. Theor. Phys. 68, 927 (1982); 70, 330 (1983); L. E. Ibanez, Phys. Lett. 118B, 73 (1982); J. R. Ellis, D. V. Nanopoulos, and K. Tamvakis, Phys. Lett. 121B, 123 (1983); L. Alvarez-Gaume, J. Polchinski, and M. B. Wise, Nucl. Phys. B221, 495 (1983).

[44] K. Nakamura et al. (Particle Data Group), J. Phys. G 37, 075021 (2010).

[45] H. Baer and M. Brhlik, Phys. Rev. D 55, 4463 (1997); H. Baer, M. Brhlik, D. Castano, and X. Tata, Phys. Rev. D 58, 015007 (1998).

[46] K. Babu and C. Kolda, Phys. Rev. Lett. 84, 228 (2000); A. Dedes, H. Dreiner, and U. Nierste, Phys. Rev. Lett. 87, 251804 (2001); J. K. Mizukoshi, X. Tata, and Y. Wang, Phys. Rev. D 66, 115003 (2002).

[47] RAaij et al. (LHCb Collaboration), Phys. Rev. Lett. 110, 021801 (2013).

[48] Y. Amhis et al. (Heavy Flavor Averaging Group Collaboration), arXiv:1207.1158.

[49] D. Asner et al. (Heavy Flavor Averaging Group Collaboration), arXiv:1010.1589.

[50] G. Hinshaw et al. (WMAP Collaboration), arXiv:1212.5226.

[51] B. He, T. Li, and Q. Shafi, J. High Energy Phys. 05 (2012) 148.

[52] H. Baer and X. Tata, Weak Scale Supersymmetry: From Superfields to Scattering Events (Cambridge University Press, Cambridge, England, 2006), p. 537.

[53] M. Kawasaki, K. Kohri, T. Moroi, and A. Yotsuyanagi, Phys. Rev. D 78, 065011 (2008).

[54] J. Pradler and F. D. Steffen, Phys. Lett. B 666, 181 (2008); D. G. Cerdeno, K.-Y. Choi, K. Jedamzik, L. Roszkowski, and R. Ruiz de Austri, J. Cosmol. Astropart. Phys. 06 (2006) 005; L. Roszkowski, R. Ruiz de Austri, and K.-Y. Choi, J. High Energy Phys. 08 (2005) 080.

[55] See, incomplete set of references, J. R. Ellis, K. A. Olive, and Y. Santoso, J. High Energy Phys. 10 (2008) 005; M. Kawasaki, K. Kohri, and T. Moroi, Phys. Lett. B 649, 436 (2007); J. L. Diaz-Cruz, J. R. Ellis, K. A. Olive, and Y. Santoso, J. High Energy Phys. 05 (2007) 003; R. H. Cyburt, J. R. Ellis, B. D. Fields, K. A. Olive, and V.C. Spanos, J. Cosmol. Astropart. Phys. 11 (2006) 014; T. Kanzaki, 
M. Kawasaki, K. Kohri, and T. Moroi, Phys. Rev. D 75, 025011 (2007); F. D. Steffen, J. Cosmol. Astropart. Phys. 03 (2006) 001; J. L. Feng, S. Su, and F. Takayama, Phys. Rev. D 70, 075019 (2004); H. K. Dreiner, M. Hanussek, J. S. Kim, and S. Sarkar, Phys. Rev. D 85, 065027 (2012).

[56] J. L. Diaz-Cruz, J. R. Ellis, K. A. Olive, and Y. Santoso, J. High Energy Phys. 05 (2007) 003.
[57] G. F. Giudice and R. Rattazzi, Phys. Rep. 322, 419 (1999).

[58] S. Dimopoulos, S. D. Thomas, and J. D. Wells, Nucl. Phys. B488, 39 (1997).

[59] L. Covi and F. Dradi, J. Cosmol. Astropart. Phys. 10 (2014) 039. 